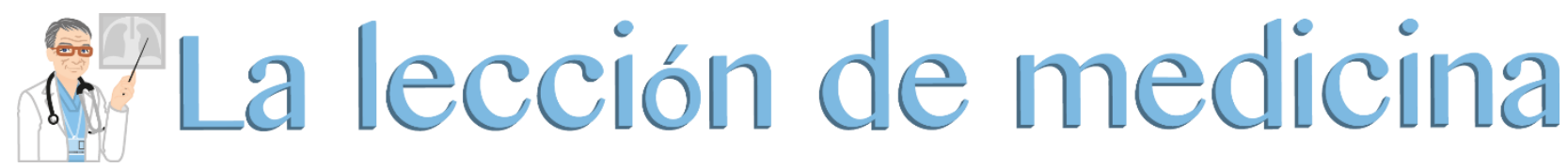

\title{
ENFERMEDAD CORONARIA BASADA EN LA EUIDENGIA
}

Gonzalo Bernal F. ${ }^{+++++}$

\section{INTRODUCCIÓN}

Aprender y entender, son conceptos bien diferentes. Entender determina comprender, incluso asimilar conceptos pero, de ahí, a aprender, existe una gran distancia. Aprender es aplicar lo que se entiende, es tomar el concepto y usarlo para la vida.

Este documento forma parte de la sección que hemos denominado La lección de Medicina. Una vez más, el profesor Bernal nos ha construido un hermoso material donde usa buena parte de su capacidad didáctica para hacernos entender, en esta ocasión, conceptos básicos acerca de la enfermedad coronaria. Nos pone de manifiesto cómo se revisa y se reconoce el origen de las fuentes. Nos ayuda a entender una serie de conceptos elementales en torno al dolor torácico y nos expone una sencilla clasificación. Nos hace entender la importancia de los diagnósticos diferenciales, usando precisamente como ejemplo dicho dolor. Nos introduce en las anginas torácicas y en los movimientos enzimáticos cuando las coronarias obstruidas resienten al miocardio, todo para resaltar las manifestaciones más frecuentes, vistas bajo la sensibilidad y la especificad a través de los cual nos invita a hacer diagnósticos más precisos, acercándonos a ese maravilloso mundo de la Medicina Basada en la Evidencia. Nos pareció que puso toda su pericia didáctica y la concretó en este maravilloso ejemplo.

Esperamos que ustedes, nuestros lectores, entiendan esta lección y luego la apliquen a otras áreas. Con el fin de orientar su proceso de aprendizaje, se harán algunas preguntas. Les pedimos que al responderlas, pongan la referencia de donde obtuvieron la respuesta. Se trata de una de las habilidades que debemos "rutinizar" en todo proceso de revisión, ¿no les parece?

Como siempre, ibienvenidos y feliz aprendizaje!

\section{Contexto}

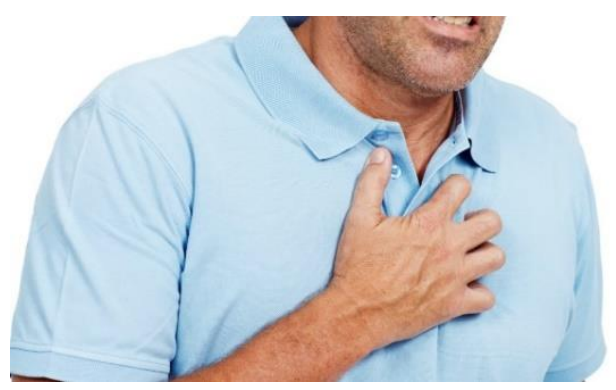

En la consulta médica de los servicios de urgencias, el dolor torácico es una causa frecuente de la cual se derivan patologías como la enfermedad coronaria. Las enfermedades cardiovasculares siguen siendo la principal causa de muerte en el mundo (1). Según estadísticas de la American Heart Association, en Estados Unidos 17,6 millones de personas tienen enfermedad coronaria. The Global Burden of Disease Study 2013 estima que 17,3 millones de muertes en el mundo se relacionan con enfermedad coronaria. Según el estudio de Framingham la incidencia de eventos ateromatosos en la población de 35 a 64 años se presenta con una frecuencia de 12 hombres y 5 mujeres por cada 1000 personas, respectivamente, $y$, en las edades de 65 años a 94 años, se presentan 27 casos en hombres contra 16 en mujeres por cada 100.000 personas.

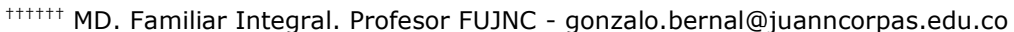




\section{Nosotros}

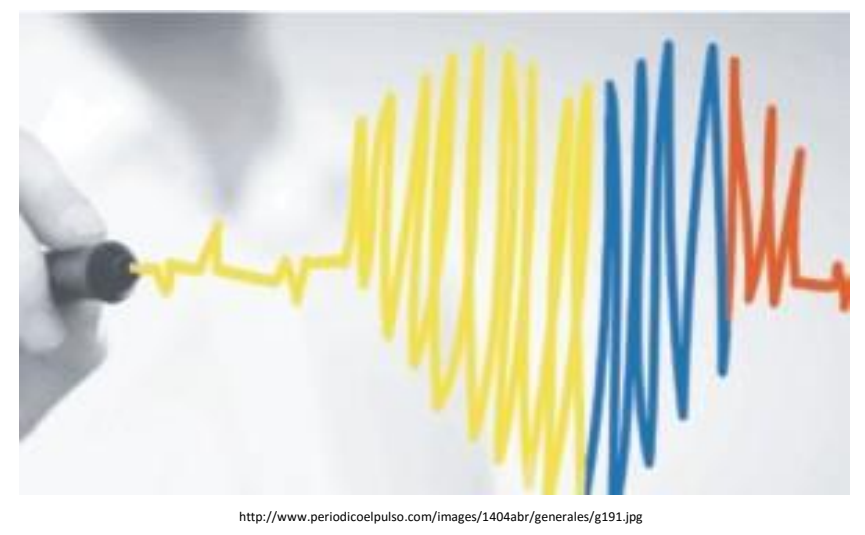

En Colombia, después de la violencia, las causas cardiovasculares continúan siendo la primera causa de mortalidad (23,5\% de todas las causas de muertes del país) y de este porcentaje:

- El 56,3\% corresponde a cardiopatía isquémica.

- El 30,6 \% a eventos cerebrovasculares.

- El 12,4\% a enfermedad hipertensiva.

- El 0,5\% a enfermedad cardiaca de origen reumático.

Los departamentos de Tolima, Caldas, Quindío, Risaralda, San Andrés y Providencia, Norte de Santander, Huila, Valle del Cauca y Antioquia evidenciaron las mayores tasas de mortalidad ajustadas por edad $(2,3)$.

\section{Factores de riesgo}

Según la Revista Colombiana de Cardiología, en su publicación de 2008, los porcentajes de la prevalencia de los factores de riesgo para enfermedades cardiovasculares en Colombia son los siguientes:

1. Sedentarismo en adolescentes de 13 a 17 años con 27,6 $\%$ en hombres y $24,2 \%$ en mujeres.

2. Sedentarismo en adultos entre 18 y 64 años con $38,1 \%$ en hombres y $46,4 \%$ en mujeres.

3. Tabaquismo con $26,8 \%$ en hombres y $11,3 \%$ en mujeres.
4. Hipertensión arterial informada por el paciente con 11,5 $\%$ en hombres y $13 \%$ en mujeres.

5. Diabetes mellitus tipo 2 con $1,3 \%$ en hombres y $2,6 \%$ en mujeres.

6. Sobrepeso en adultos entre 18 y 64 años con $31,1 \%$ en hombres y $33 \%$ en mujeres.

6.1. Obesidad en adultos entre 18 y 64 años con $8,8 \%$ en hombres y $16,6 \%$ en mujeres.

\section{Evolución del dolor torácico}

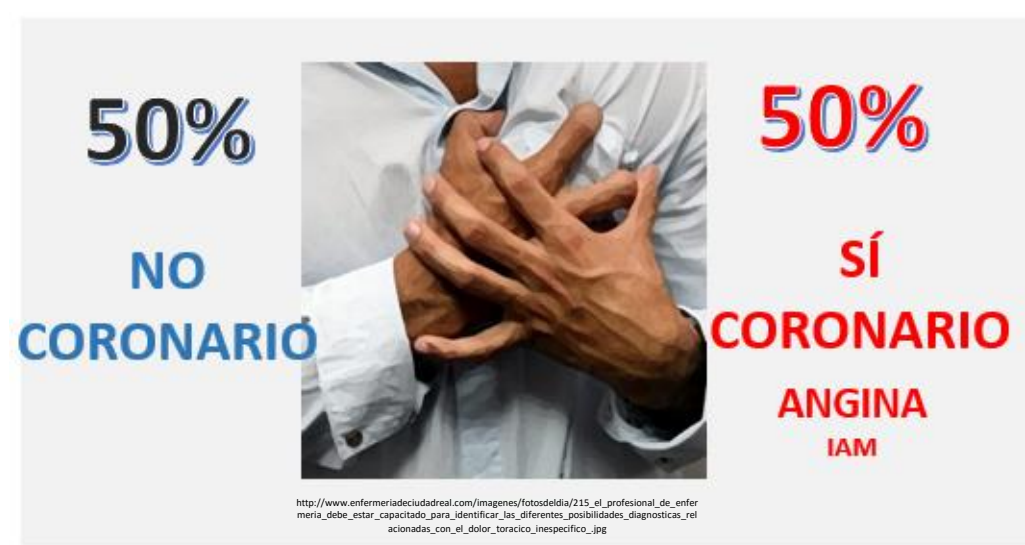

Según un estudio publicado en la revista New England Journal of Medicine (4), cinco millones de personas acuden por dolor torácico a los servicios de urgencias en Estados unidos y de ellos:

$-4{ }^{2}$ El $50 \%$ se hospitaliza y de estos:

- Solo el $15 \%$ presenta un infarto agudo de miocardio.

- Aproximadamente el $30 \%$ presenta una angina inestable (4).
La prevalencia en Europa de enfermedad coronaria oscila entre el $14 \%$ y el $24 \%$ con el dolor típico (5).

Reconociendo que actualmente hay bastante información a nuestra disposición, el presente módulo pretende revisar la enfermedad coronaria aguda; así como los conceptos de sensibilidad, especificidad, valores predictivos y coeficientes de verosimilitud y su aplicación en la práctica clínica. 


\section{ENFOQUE DIAGNÓSTICO DEL PACIENTE CON DOLOR TORÁCICO Y PROBABLE ENFERMEDAD CORONARIA}

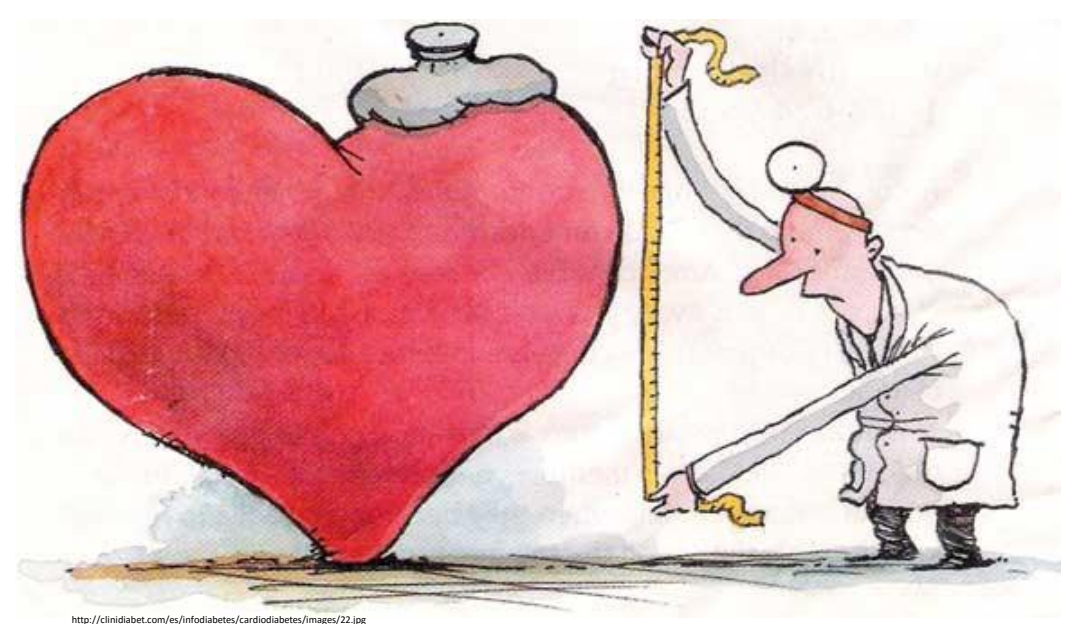

Un paciente con enfermedad coronaria puede consultar por presentar dolor torácico o por algunas de sus complicaciones o equivalentes.

\section{Dolor torácico}

Si un paciente ingresa por dolor torácico puede presentar alguna de estas probabilidades:

\section{Dolor torácico de origen cardiogénico}

Este puede dividirse en dos grandes grupos:

a. Coronario.

b. No coronario.

\section{a. Cardiaco de origen coronario}

El dolor torácico de origen coronario se subdivide a su vez en tres grupos:

-k $\Omega_{r}$ Infarto agudo de miocardio con elevación del ST.

- Infarto agudo de miocardio sin elevación del ST.

Has Angina inestable.

\section{b. No coronario}

El dolor torácico no coronario se puede deber a patologías como:

-

$-4 \Omega_{2}$ Miocarditis.

- Nar Disección de la aorta.

$-\Omega_{2}$ Estenosis aórtica.

- Insuficiencia aórtica.

- Estenosis mitral. 


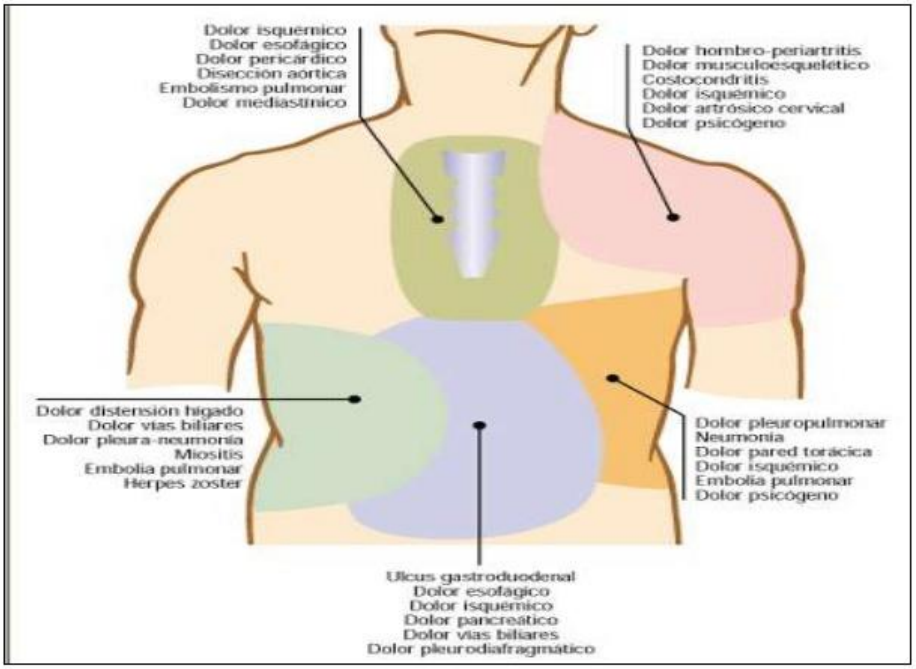

\section{Dolor torácico de origen no cardiaco}

Este puede ser secundario a causas cuyo origen no corresponden al corazón, como son las de origen:

$-4 \Omega_{-}$Pulmonar (neumonía, pleuritis, tromboembolismo pulmonar, etc.).

$-\mu \Omega_{-}$Digestivo (pancreatitis, enfermedad de la vía biliar, enfermedades del estómago como el ulcus péptico, etc.)

$-1 \Omega_{-}$Neurológico (herpes, neuropatías periféricas, compresiones radiculares, etc.).

$-1 \Omega_{r}$ Osteomuscular (trauma, espondilitis anquilosante, etc.).

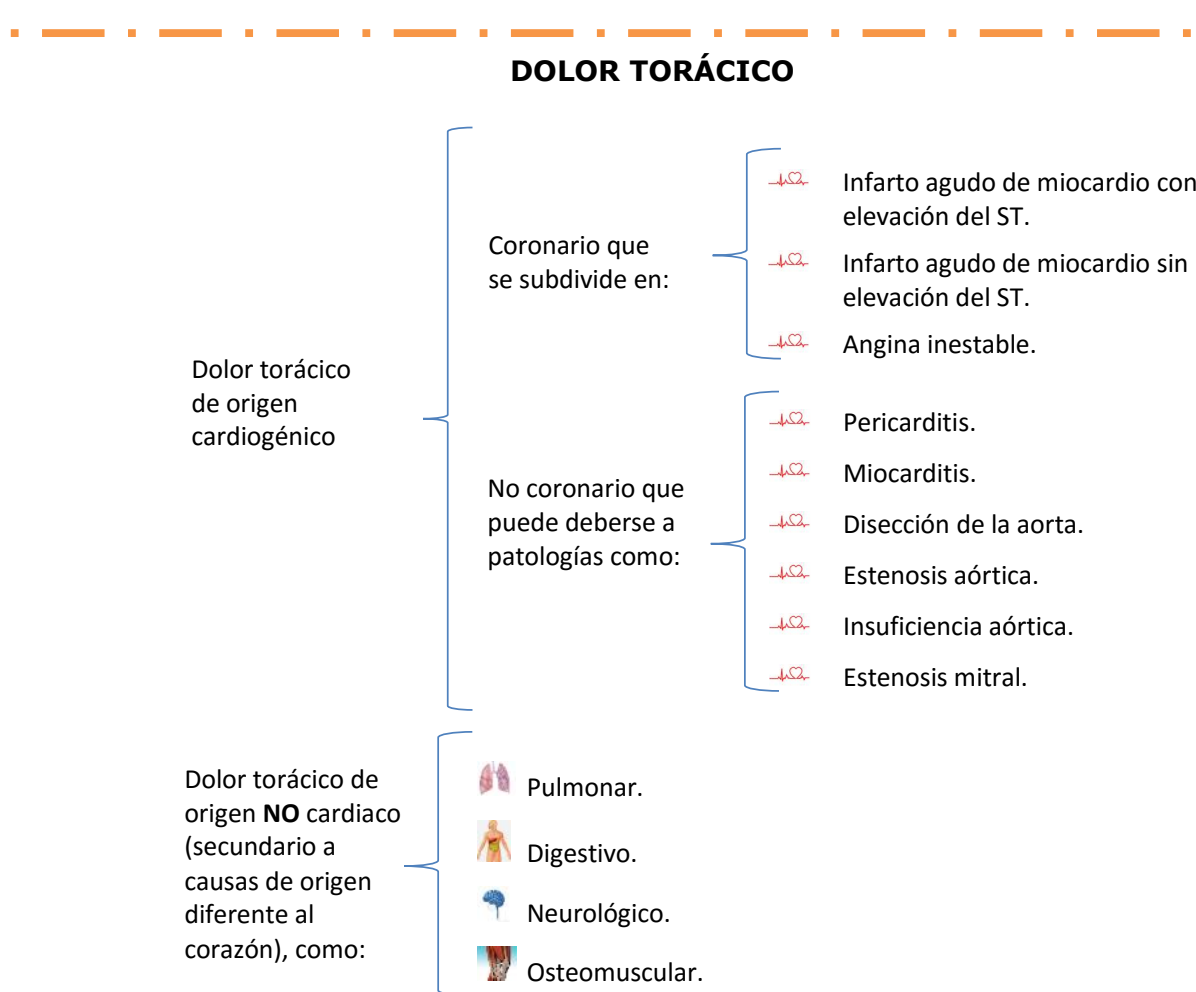




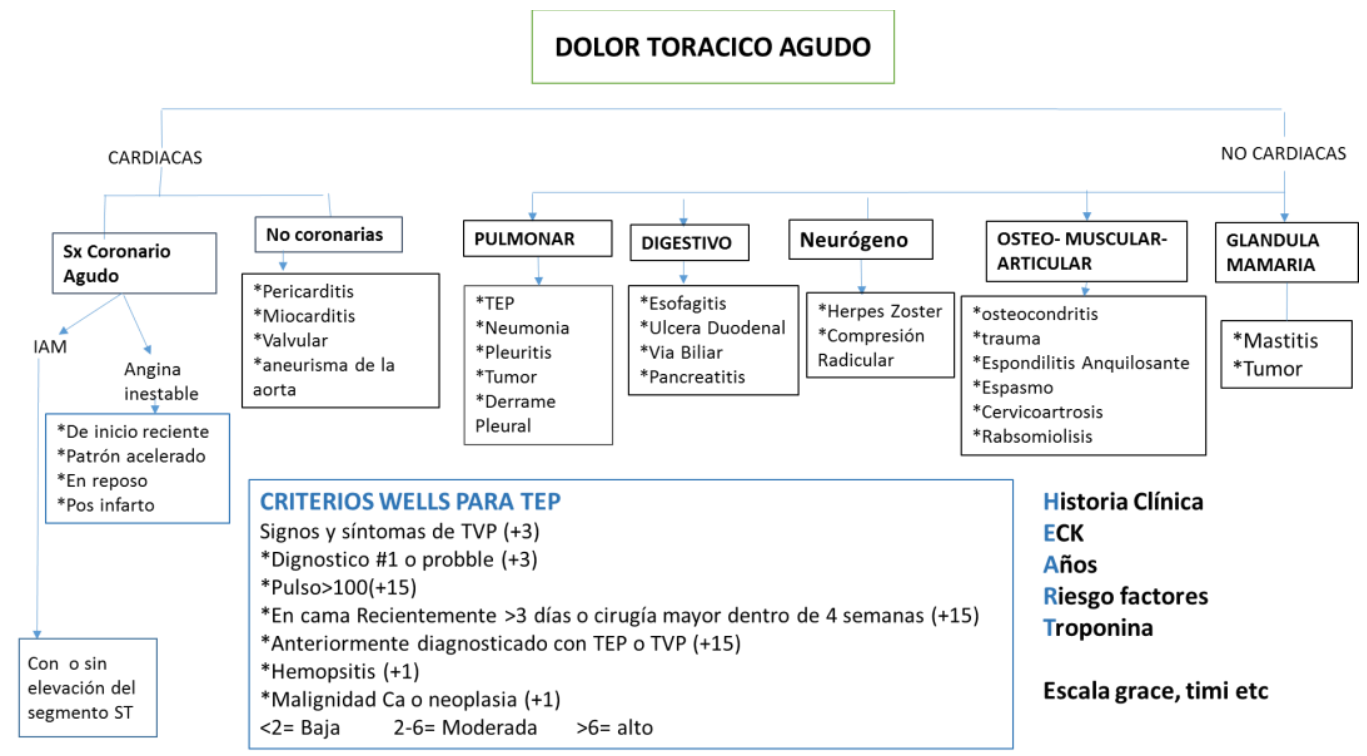

\section{Otras presentaciones del paciente coronario}

Puede asistir por manifestaciones equivalentes o complicaciones $(6,7)$. Es decir, el paciente puede presentar un evento coronario, pero manifestado por:

a. Disnea, que correspondería a una angina equivalente al $16 \%$.

b. Arritmias.

c. Evento cerebrovascular.

d. Falla cardiaca. e. Muerte súbita.

f. Síncope.

g. Dolor abdominal (principalmente en pacientes con diabetes, ancianos con demencia senil o falla renal) (7).

\section{Definiciones}

Las siguientes definiciones están basadas en los criterios diagnósticos de Patterson y Morowitz para angina estable (8, 9. 10).

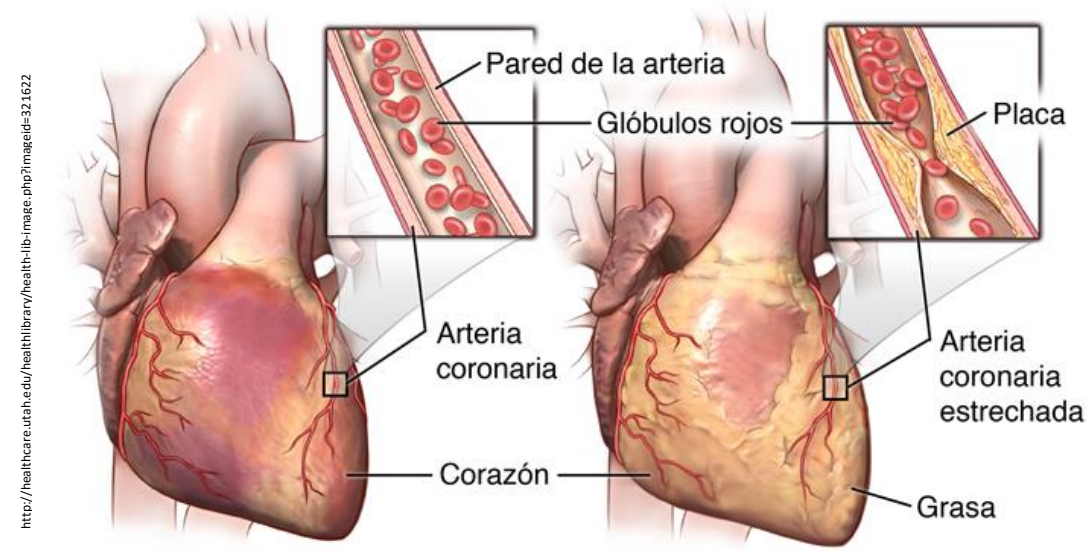

Corazón y la arteria normal Arteria con la acumulación de placa 
1. Angina típica (definitiva). Compuesta por:

1.1. Si es un dolor subesternal de características y duración coronaria.

1.2. Provocado por ejercicio o estrés emocional.

1.3. Aliviado con reposo o nitroglicerina.

2. Angina atípica (probable): reúne dos de las características anteriores.

3. Dolor torácico no cardíaco: reúne una o ninguna de las características de la angina típica.

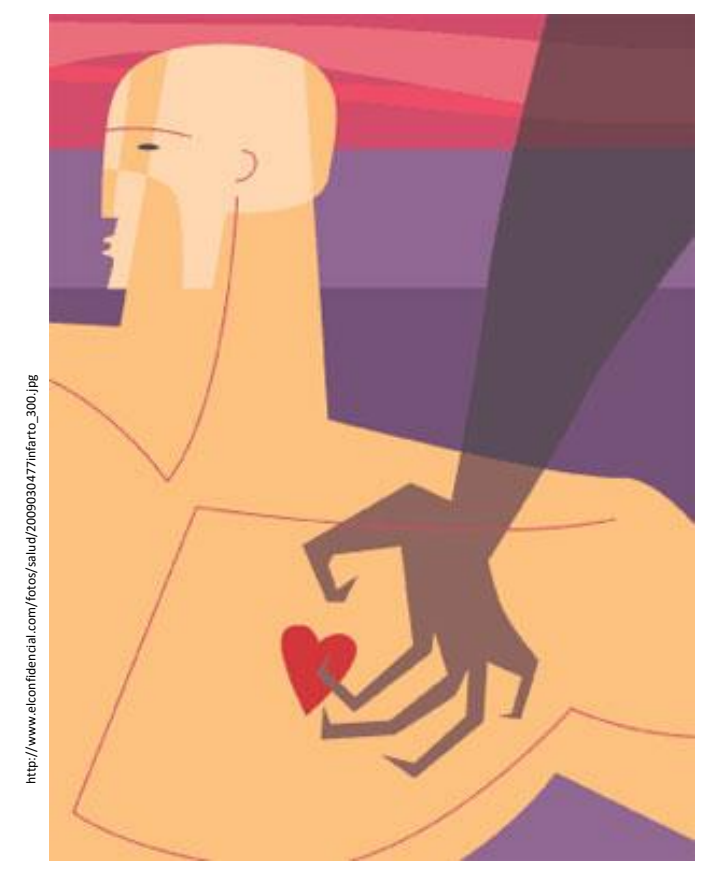

4. Angina inestable: corresponde a un espectro de enfermedades que van entre el infarto agudo de miocardio y la angina estable. La Sociedad Europea de Cardiología la define en sus guías de 2015 (11) como la isquemia miocárdica que se presenta en el reposo o con mínimo esfuerzo en ausencia de necrosis. Se divide en forma general en cuatro tipos:

4.1. Angina de patrón acelerado o in crescendo: se caracteriza por un cambio en el comportamiento de un síntoma crónico en lo referente a la intensidad, duración y desencadenamiento de la isquemia.

4.2. Angina de reciente inicio: es aquella que se presenta con un tiempo de aparición menor de dos meses, de acuerdo a la clasificación canadiense de angina.

4.3. Angina de reposo: se presenta con el reposo agudo o subagudo en menos de 48 horas y con una duración de 20 minutos o más.

4.4. Angina postinfarto: es la que se presenta después de 15 días de haber presentado un infarto agudo de miocardio. 


\section{Definición de infarto agudo de miocardio (IAM) $(11,12)$}

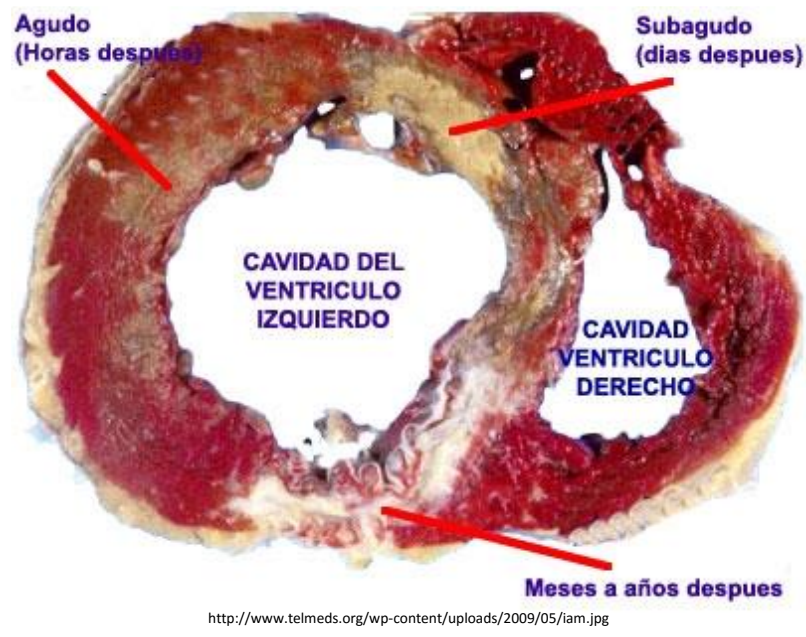

El término IAM se debe utilizar cuando haya pruebas de necrosis miocárdica en un contexto clínico coherente con isquemia miocárdica aguda. En esas condiciones, cualquiera de los criterios siguientes cumple el diagnóstico de IAM:

\section{A partir de enzimas}

\section{X por encima de la normalidad}
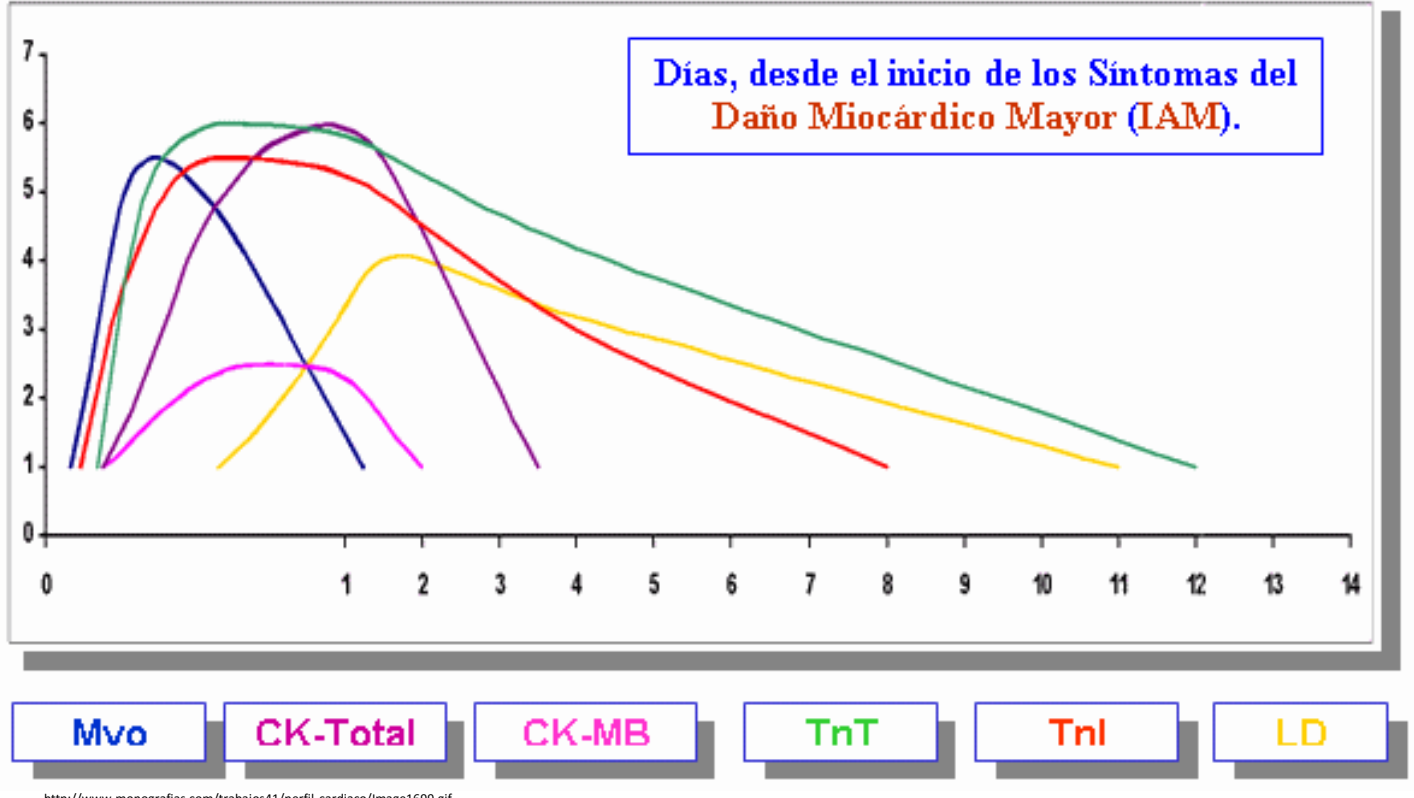

Detección de un aumento o descenso de los valores de biomarcadores cardiacos (preferiblemente cTn) con al menos un valor por encima del p99 del LRS (límite superior) y con al menos uno de los siguientes: 
Hôr Síntomas de isquemia.

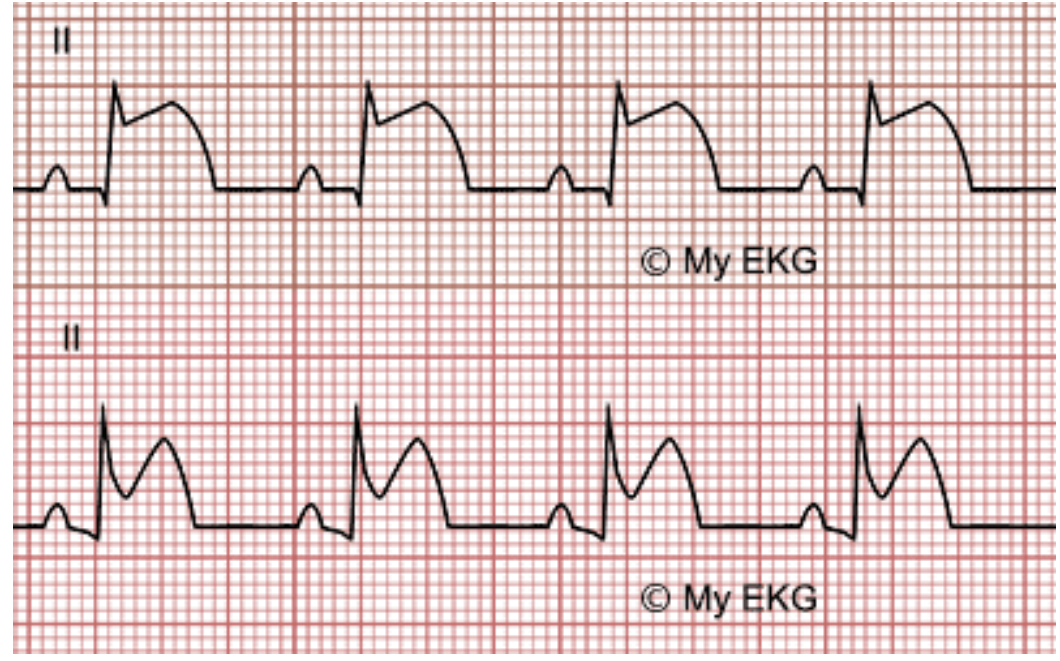

http://www.my-ekg.com/imag/difierencia-iamest-pericarditis.png

H Ner Nuevos o supuestamente nuevos cambios significativos del segmento ST-T o nuevo BRIHH (bloqueo de rama izquierda del haz de hiss).

Har Aparición de ondas Q patológicas en el ECG (electrocardiograma).

la pared.

Her Identificación de un trombo intracoronario en la angiografía o la autopsia.

- w $\Omega_{2}$ Muerte cardiaca con síntomas de isquemia miocárdica y supuestas nuevas alteraciones isquémicas en el ECG o nuevo BRIHH, pero que se produjo antes de determinar.

Hor Biomarcadores cardiacos o antes de que aumentasen los valores de estos.

\section{A partir de angiografía}

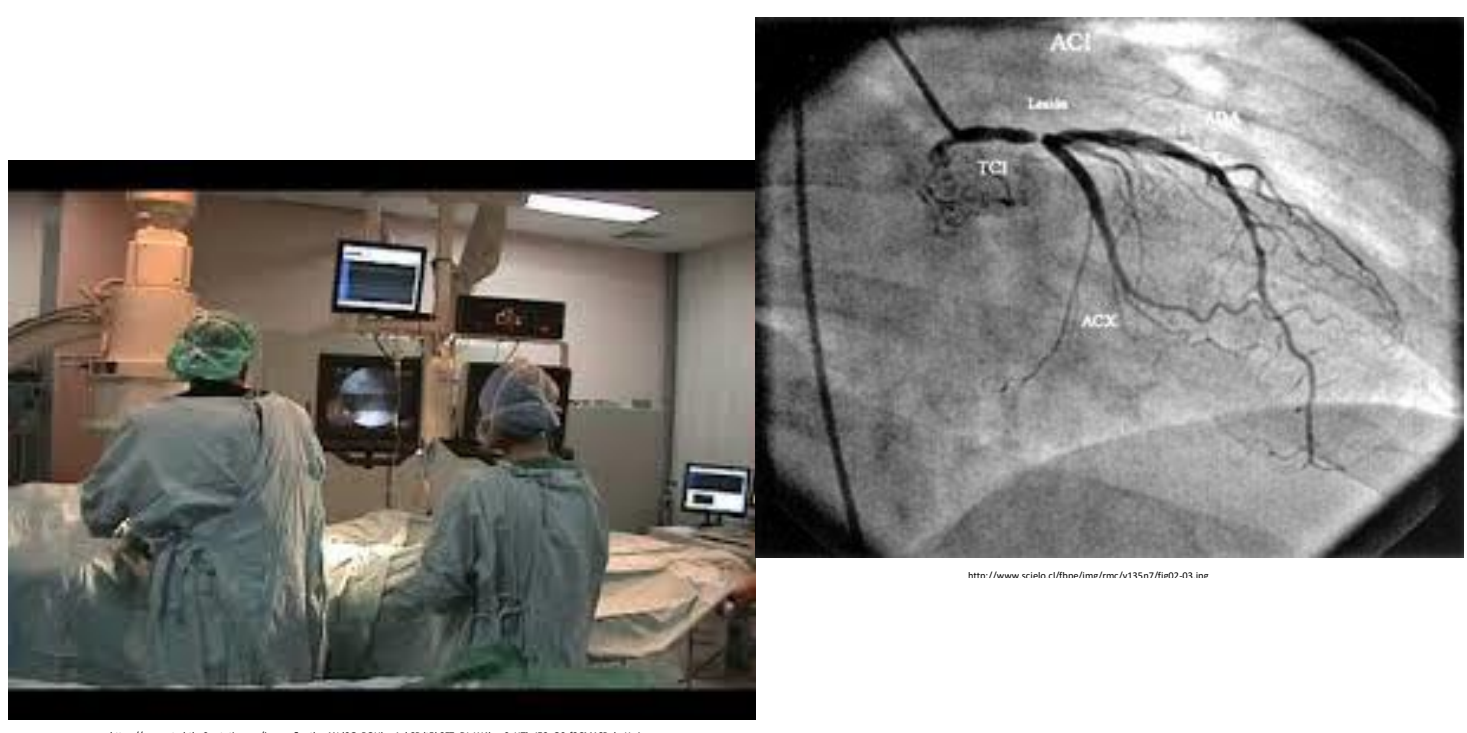


Se define arbitrariamente el IAM relacionado con ICP (intervención coronaria percutánea o angioplastia percutánea) por:

La elevación de cTn (troponina) (> $5 \times$ p99 del LRS) en pacientes con valores basales normales $(\leq \mathrm{p} 99$ del LRS).

O, un aumento de los valores de cTn > $20 \%$ si los basales son elevados y estables o descienden.

Además, se necesita:

Síntomas de isquemia miocárdica.

Nuevos cambios isquémicos del ECG.

Hallazgos angiográficos coherentes con complicación del procedimiento.
- war Demostración por imagen de nueva pérdida de miocardio viable o nuevas anomalías regionales en el movimiento de la pared.

$-4 \Omega_{r}$ La trombosis del stent asociada a IM, si se detecta en la angiografía coronaria o la autopsia en el contexto de isquemia miocárdica y con un aumento o descenso de los títulos de biomarcadores cardiacos con al menos un valor > p99 del LRS.

\section{Relacionado con la cirugía de revascularización}

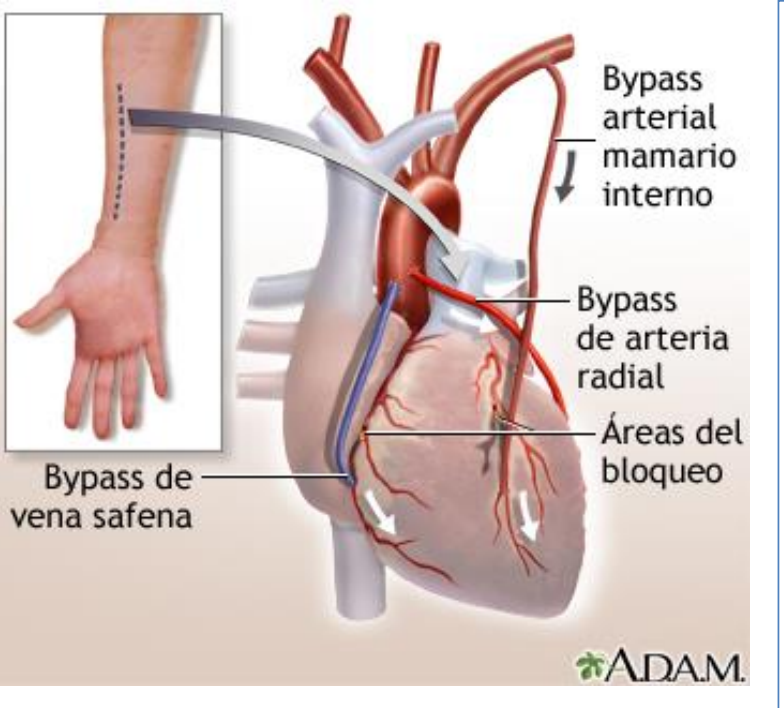

EI IAM relacionado con la CABG (cirugía de revascularización cardiaca) se define arbitrariamente por:

- La elevación de títulos de biomarcadores cardiacos (> $10 \times$ p99 del LRS) en pacientes con valores basales de cTn normales ( $\leq$ p99 del LRS).

- Además, se debe considerar diagnóstico de IM nuevas ondas Q patológicas o nuevo BRIHH.

- Nuevo injerto documentado angiográficamente o nueva oclusión de la arteria coronaria nativa.

- pruebas por imagen de nueva pérdida de miocardio viable o nuevas anomalías regionales en el movimiento de la pared (12).

\section{Abordaje del paciente}

\section{$\rightarrow$ De entrada}

Para iniciar el abordaje del paciente con dolor torácico se debe realizar:

1. Una adecuada historia clínica.

2. Un electrocardiograma.

3. Tomar biomarcadores. 


\section{$\rightarrow$ La historia clínica}

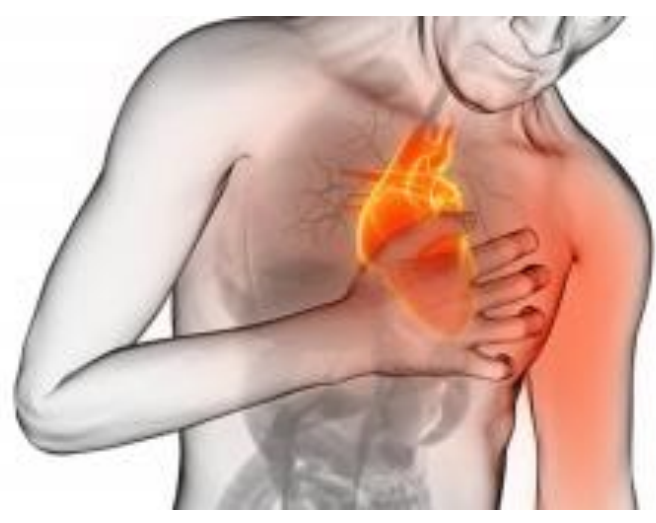

Debe hacer énfasis en la semiología del dolor torácico buscando características como:

-4nßr Localización, intensidad, duración, frecuencia.

-tws Qué actividades lo aumentan, qué actividades o medicamentos lo disminuyen.

-w准 Qué síntomas asociados presenta, buscar principalmente cortejo neurovegetativo, etc.

Además, se identificarán:

- ther Factores de riesgo como tabaquismo, sedentarismo, hiperlipidemia, diabetes, etc.

$\rightarrow \mathrm{A}_{\mathrm{r}}$ Datos relevantes al examen físico como los signos vitales (cambios de la tensión arterial), galope por S3, signos de falla cardiaca, etc.

Tabla 1. Características del dolor torácico

\begin{tabular}{|c|c|c|c|c|c|c|c|c|c|c|}
\hline & TIPO & CARÁCTER & LUGAR & INICIO & IRRADIA & DURACIÓN & APARICIÓN & ASOCIADO & MEJORÍA & AGRAVA \\
\hline ANGINA & Isquémico. & Opresivo. & Retroesternal. & Brusco. & $\begin{array}{c}\text { Hombre, brazo, } \\
\text { mandibula. }\end{array}$ & $\begin{array}{l}\text { De } 2 \text { a } 20 \\
\text { minutos. }\end{array}$ & Progresivo/súbito. & $\begin{array}{l}\text { Sintomas } \\
\text { vagales. }\end{array}$ & $\begin{array}{l}\text { Reposoo } \\
\text { nitrittos. }\end{array}$ & Esfuerzo. \\
\hline INFARTO & Isquémico. & Opresivo. & Retroesternal. & Brusco. & $\begin{array}{c}\text { Hombre, brazo, } \\
\text { mandibula. }\end{array}$ & $\begin{array}{l}\text { Más de } 20 \\
\text { minutos. }\end{array}$ & Progresivo/súbito. & $\begin{array}{c}\text { Sintomas } \\
\text { vagales. }\end{array}$ & & Esfuerzo. \\
\hline ANEURISMA & Isquémico. & Lancinante. & Retroesternal. & Brusco. & $\begin{array}{c}\text { Hombre, brazo, } \\
\text { mandibula. }\end{array}$ & $\begin{array}{l}\text { Más de } 20 \\
\text { minutos. }\end{array}$ & Progresivo/súbito. & Neorológicos. & & Esfuerzo. \\
\hline MEDIASTINITIS & Pleurítico. & Lancinante. & Retroesternal. & Brusco. & Costado. & Prolongado. & Progresivo/súbito. & Fiebre. & & Movimiento. \\
\hline PERICARDITIS & Pleurítico. & Opresivo/pleurítico. & Retroesternal. & Insidioso. & Costado. & Prolongado. & Progresivo/súbito. & $\begin{array}{c}\text { Fiebre. } \\
\text { Cambios de } \\
\text { posición. }\end{array}$ & $\begin{array}{c}\text { Inclinación } \\
\text { hacia } \\
\text { adelante. }\end{array}$ & $\begin{array}{c}\text { Tos, } \\
\text { inspiración. }\end{array}$ \\
\hline $\begin{array}{l}\text { EMBOLISMO } \\
\text { PULMONAR }\end{array}$ & Pleurítico. & Variable. & Variable. & Variable. & $\begin{array}{c}\text { Costado } \\
\text { (variable). }\end{array}$ & Prolongado. & Progresivo/súbito. & $\begin{array}{c}\text { Sintomas } \\
\text { inespecificos. }\end{array}$ & & $\begin{array}{c}\text { Tos, } \\
\text { inspiración. }\end{array}$ \\
\hline NEUMONÍA & Pleurítico. & Variable. & Variable. & Variable. & Variable. & Prolongado. & Progresivo/insidioso. & Fiebre. & & $\begin{array}{c}\text { Tos, } \\
\text { inspiración. }\end{array}$ \\
\hline NEUMOTÓRAX & Pleurítico. & Costado. & Costado. & Brusco. & Costado, hombro. & Prolongado. & Progresivo/insidioso. & $\cdot$ & Reposo. & $\begin{array}{c}\text { Tos, } \\
\text { inspiración. }\end{array}$ \\
\hline
\end{tabular}




\section{¿CUÁLES SON LOS SIGNOS Y SÍNTOMAS MÁS IMPORTANTES EN LA ENFERMEDAD CORONARIA?}

\section{JAMAevidence}

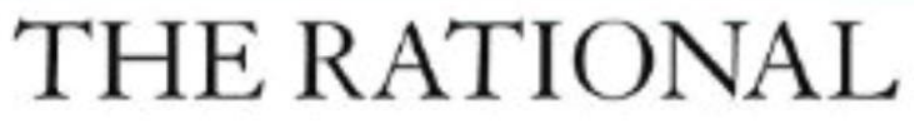

CLINICAL

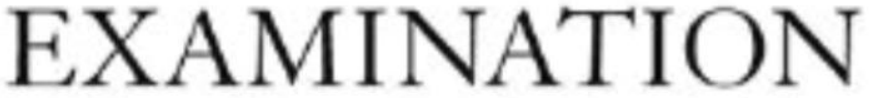

\section{EVIDENCE-BASED CLINICAL DIAGNOSIS}

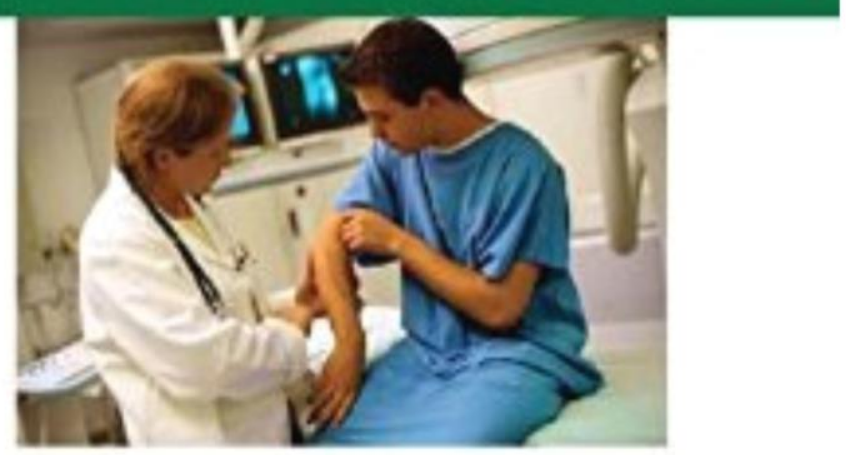

David L. Simel, MD, MHS - Drummond Rennic, MD

La revista JAMA en su publicación de The Rational Clinical Examination nos muestra los diferentes estudios con sus correspondientes sensibilidades, especificidades y coeficientes de verosimilitud (tabla 2) tanto de los factores de riesgo, como los síntomas y la evaluación del electrocardiograma (13).

\section{Lo típico}

De forma clásica los pacientes se presentan con dolor retro esternal opresivo irradiado a brazos y cuello, o dolor en abdomen, asociado a síntomas vágales como astenia, náuseas, vómito y diaforesis.

\section{Pero...}

Según Framingham, el $25 \%$ de los pacientes no son reconocidos, por no presentar este patrón o ni siquiera dolor. Es por esto que el dolor torácico siempre ha sido un reto médico en el que, en muchas ocasiones, nos es difícil establecer qué tipo de acciones tomar y llevarnos incluso a errores como enviar a casa (entre el 10 al $15 \%$ ) pacientes con enfermedad coronaria aguda. 
Tabla 2. Sensibilidad, especificidad y LR de factores de riesgo, síntomas y electrocardiograma en la enfermedad coronaria

\begin{tabular}{|c|c|c|c|c|c|}
\hline CARACTERISTICA & SENSIBILIDAD & ESPECIFICIDAD & LR(+) & LR (-) & BIBLIOGRAFIA \\
\hline \multicolumn{6}{|l|}{$\begin{array}{l}\text { FACTORES DE } \\
\text { RIESGO }\end{array}$} \\
\hline ESTRÉS & $12(8.3-16)$ & $96(95-97)$ & $2.1(2-4.7)$ & $0.92(0.88-0.06)$ & $\begin{array}{l}\text { Nerenberg, et } \\
\text { al. } 2007\end{array}$ \\
\hline ENFERMEDAD ARTERIAL PERIFERICA & $8.3(5.6-12)$ & $96(95-96)$ & $1.9(1.2-2.8)$ & $0.96(0.93-0,99)$ & Hess et al. 2012 \\
\hline $\begin{array}{l}\text { ANTECEDENTE DE ENFERMEDAD } \\
\text { CORONARIA }\end{array}$ & $67(59-74)$ & $59(55-63)$ & $1.6(1.4-1.9)$ & $0.56(0.45-0.70)$ & $\begin{array}{l}\text { Christenson et } \\
\text { al. } 2006\end{array}$ \\
\hline DIABETES & $30(25-35)$ & $81(79-82)$ & $1.5(1.3-1.8)$ & $0.87(0.81-0.94)$ & Hess et al.2012 \\
\hline ECV & $10(7.4-14)$ & $94(92-95)$ & $1.6(1.1-2.3)$ & $0.96(0.92-1.0)$ & Hess et al.2012 \\
\hline HIPERLIPIDEMIA & $71(66-76)$ & $49(47-51)$ & $1.4(1.3-1.5)$ & $0.59(0.50-1)$ & Hess et al.2013 \\
\hline HIPERTENSION & $72(67-77)$ & $43(41-45)$ & $1.3(1.2-1.4)$ & $0.64(0.530 .76)$ & Hess et al.2014 \\
\hline TABAQUISMO & $57(52-63)$ & $50(48-52)$ & $1.1(1.0-1.3)$ & $0.86(0.75-0.98)$ & Hess et al.2014 \\
\hline $\begin{array}{c}\text { HISTORIA FAMILIAR DE ENFERMEDAD } \\
\text { CORONARIA }\end{array}$ & $28(24-33)$ & $71(69-73)$ & $0.98(0.82-1.2)$ & $1.0(0.94-1.0)$ & Hess et al.2014 \\
\hline OBESIDAD & $17(5.6-35)$ & $89(85-92)$ & \begin{tabular}{|l|}
$1.5(0.64-3.5)$ \\
\end{tabular} & $0.94(0.80-1.1)$ & Melki et al.2013 \\
\hline $\begin{array}{l}\text { PREVIA REVASCULARIZACION } \\
\text { MIOCARDICA }\end{array}$ & $13(8.5-18)$ & $93(92-94)$ & $1.8(1.3-2.7)$ & $0.94(0.89-1.0)$ & McDonald etal.2011 \\
\hline \multirow[t]{2}{*}{ SINTOMAS } & & & & & \\
\hline & SENSIBILIDAD & ESPECIFICIDAD & $\mathbf{L R}(+)$ & LR (-) & BIBLIOGRAFIA \\
\hline IIRADIACION A AMBOS BRAZOS & $11(8.3-15)$ & $96(95-96)$ & $2.6(1.8-3.7)$ & $0.93(0.89-0.96)$ & Hess et al.,2012 \\
\hline DOLOR PARECIDO AL ANGINOSO & $47(42-53)$ & $79(77-80)$ & $2.2(2.0-2.6)$ & $0.67(0.60-0.74)$ & Hess et al.,2012 \\
\hline $\begin{array}{c}\text { CAMBIO DEL PATRON DEL DOLOR EN } \\
48 \text { HORAS }\end{array}$ & $27(23-32)$ & $86(85-88)$ & $2.0(1.6-2.5)$ & $0.84(0.79-0.90)$ & Hess et al.,2012 \\
\hline DOLOR PRECORDIAL TIPICO & $73(67-78)$ & 89 (86-92) & $6.8(5.1-9.2)$ & $0.30(0.25-0.37)$ & Biener et al.2015 \\
\hline AUMENTO DEL DOLOR CON EJERCICIO & $53(48-59)$ & $71(69-73)$ & $1.8(1.6-2.1)$ & $0.66(0.58-0.74)$ & Hess et al.,2012 \\
\hline IRRADIACION A CUELLO Y MANDIBULA & $24(19-28)$ & $85(83-86)$ & $1.6(1.3-1.9)$ & $0.90(0.85-0.96)$ & Hess et al.,2012 \\
\hline $\begin{array}{c}\text { episodios reciente y similares de } \\
\text { dolor toracico }\end{array}$ & $55(50-60)$ & $56(54-59)$ & $1.3(1.1-1.4)$ & $0.80(0.71-0.90)$ & Van Der Meer et al.2015 \\
\hline irradiacion a brazo izquierdo & $5.4(3.4-8.3)$ & $96(95-97)$ & $1.3(0.78-2.1)$ & $0.99(0.96-1.0)$ & Hess et al.,2012 \\
\hline irradiacion a brazo derecho & $5.4(3.4-8.3)$ & $96(95-97)$ & 1.3(0.78-2.1) & $0.99(0.96-2.1)$ & Hess et al.,2012 \\
\hline presencia de diaforesis & $28(24-33)$ & $79(78-81)$ & 1.4(1.1-1.6) & $0.91(0.84-0.97)$ & Hess et al.,2012 \\
\hline presencia de disnea & $47(42-53)$ & $61(59-63)$ & $1.2(1.1-1.4)$ & $0.87(0.78-0.96)$ & Hess et al.,2012 \\
\hline mejoria con nitroglicerina & $80(71-86)$ & $19(15-22)$ & 0.98(0.88-1.1) & $1.1(0.75-1.6)$ & Diercks et al.2005 \\
\hline dolor tipo ardor & $12(8.4-16)$ & $92(90-93)$ & $1.4(0.99-1.9)$ & $0.97(0.93-1.0)$ & Hess et al.,2012 \\
\hline $\begin{array}{c}\text { presencia de nausea y } \\
\text { vomito }\end{array}$ & $22(17-28)$ & $80(75-84)$ & $1.1(0.77-1.5)$ & $0.98(0.90-1.1)$ & Milner et al.2001 \\
\hline asociado a palpitaciones & $6.0(3.5-10)$ & $91(88-94)$ & $0.71(0.37-1.3)$ & $1.0(0.98-1.1)$ & Milner et al.2001 \\
\hline presencia de sincope & $6.0(3.5-10)$ & $91(88-94)$ & $0.71(0.37-1.3)$ & $1.0(0.98-1.1)$ & Milner et al.2001 \\
\hline $\begin{array}{c}\text { presencia de dolor } \\
\text { pleuritico }\end{array}$ & $6.6(4.2-9.8)$ & $82(80-83)$ & $0.14(0.10-0.19$ & $2.5(2.1-2.8)$ & Christenson et al. \\
\hline presencia de hipotension & $3.1(1.2-7.9)$ & $99(98-100)$ & $3.9(0.98-15)$ & $0.98(0.95-1.0)$ & Bjork et al.2006 \\
\hline presencia de estertores & $9.2(5.3-16)$ & $95(93-97)$ & $2.0(1.0-4.0)$ & $0.95(0.90-1.0)$ & Bjork et al.2006 \\
\hline taquipnea & $10(5.9-16)$ & $95(92-96)$ & $1.9(0.99-3.5)$ & $0.95(0.89-1.0)$ & Bjork et al.2006 \\
\hline taquicardia mayor a 120 & $10(5.9-16)$ & $95(92-96)$ & $1.9(0.99-3.5)$ & $0.95(0.89-$ & Bjork et al.2006 \\
\hline $\begin{array}{c}\text { reproduccion del dolor a la } \\
\text { palpacion }\end{array}$ & $5.5(2.5-10)$ & $80(77-84)$ & $0.28(0.14-.54)$ & $1.2(1.11-1.24)$ & Christenson etal. 2006 \\
\hline ELECTROCARDIOGRAMA & SENSIBILIDAD & ESPECIFICIDAD & LR(+) & LR (-) & BIBLIOGRAFIA \\
\hline Depresion del segmento ST & $17(13-22)$ & 97 (96-98) & $6.1(4.4-8.6)$ & $0.85(0.81-0.89)$ & Hess et al.2012 \\
\hline Depresion del segmento ST & $17(12-22)$ & $99(98-100)$ & $24(7.3-75)$ & $0.84(0.79-0.89)$ & Liu et al.,35 2015 \\
\hline onda T invertida & $15(11-19)$ & 94 (93-95) & $2.4(1.8-3.3)$ & $0.91(0.87-0.95)$ & Hess et al.2012 \\
\hline
\end{tabular}

\section{Por ello}

Son muchas las estrategias que se siguen investigando, para disminuir el riesgo que existe con la evaluación médica errónea. 


\section{UTILIDAD DE LA ESTADÍSTICA}

\section{¿Cómo utilizamos lo visto hasta aquí?}

\begin{tabular}{|c|c|c|c|}
\hline & Enfermo & Sano & $\begin{array}{l}S=V P /(V P+F N) \\
E=V N /(V N+F P) \\
V P P=V P /(V P+F P)\end{array}$ \\
\hline Positivo & VP & FP & $\begin{array}{l}\mathrm{VPN}=\mathrm{VN} /(\mathrm{VN}+\mathrm{FN}) \\
\mathrm{CPP}=\mathrm{S} /(1-\mathrm{E})\end{array}$ \\
\hline Negativo & FN & VN & $\begin{array}{l}\mathrm{CPN}=(1-\mathrm{S}) / \mathrm{E} \\
\mathrm{IE}=(\mathrm{VP}+\mathrm{VN}) /(\mathrm{VP}+\mathrm{VN}+\mathrm{FP}+\mathrm{FN}) \\
\mathrm{IY}=\mathrm{S}+\mathrm{E}-1\end{array}$ \\
\hline \multicolumn{4}{|c|}{$\begin{array}{l}\mathrm{S}=\text { sensibilidad; } \mathrm{E}=\text { especificidad; } \mathrm{VPP}=\text { valor predictivo positivo; } \mathrm{VPN}=\text { valor predic- } \\
\text { tivo negativo; } \mathrm{CPP}=\text { cociente de probabilidad positivo; } \mathrm{CPP}=\text { cociente de probabili- } \\
\text { dad negativo; } \mathrm{IE}=\text { índice de exactitud; } \mathrm{IY}=\text { índice de Youden. }\end{array}$} \\
\hline
\end{tabular}

Vamos a echar mano de la estadística. Para ello debemos recordar los conceptos de sensibilidad, especificidad y coeficientes de verosimilitud.

\section{$\rightarrow$ Sensibilidad}

Es la proporción de enfermos con la prueba positiva o la capacidad que tiene una prueba de detectar a los verdaderamente enfermos. Por ejemplo si una prueba es $100 \%$ sensible, quiere decir que no se le escapa ningún enfermo, pero si una prueba tiene una sensibilidad por ejemplo de $80 \%$, quiere decir que de 100 pacientes enfermos, detecta a 80 de ellos pero se le escapan 20, que vendrían a formar parte de los pacientes que tienen la enfermedad pero la prueba es negativa y a la que se llama FALSOS NEGATIVOS.

\section{Aplicando la sensibilidad al electrocardiograma (EKG):}

Por ejemplo, con el EKG, Backus et al (14) encontraron una sensibilidad del electrocardiograma para la detección de enfermedad coronaria aguda en $43 \%$ (38 \% - $48 \%$ ) y una

\section{$\rightarrow$ Especificidad}

Se refiere a la proporción de sanos con la prueba negativa o capacidad que tiene una prueba de detectar a los verdaderamente sanos.

En nuestro ejemplo del electrocardiograma, vemos que tiene una especificidad del $86 \%$ (que de 100 sanos, el EKG sale normal al menos en 86 ). especificidad del $86 \%$ ( $84 \%-88 \%$ ) con un coeficiente de verosimilitud positivo (LR+) de $3,0(2,6-3,6)$ y un coeficiente de verosimilitud negativo (LR-) de $0,67(0,61$ $0-73)$. Más adelante aclararemos en qué consisten estos dos términos.

Esto quiere decir que de cada 100 pacientes con enfermedad coronaria aguda, el electrocardiograma detecta solo a 43 y se le escaparán 57 pacientes que tienen enfermedad coronaria, siendo estos los falsos negativos (electro normal pero sí tienen enfermedad coronaria).

\section{Lo que de esto se deriva...}

Existen dos problemas serios con las pruebas. El primero es que el electrocardiograma puede diagnosticar correctamente a 86 pacientes de 100 enfermos. Esto quiere decir que se le escaparon 14 pacientes que son sanos, pero, que los catalogó como enfermos y a estos los llamaremos FALSOS POSITIVOS.

\section{Si hemos entendido, hasta aquí tenemos dos problemas:}

El primero es con la sensibilidad a la cual se le escaparan pacientes enfermos y no sabremos de sus desenlaces. El segundo problema es en cuanto a la especificidad, que catalogará pacientes como enfermos, siendo estos sanos y seguramente se someterán a procedimientos innecesarios. 


\section{El arte de diagnosticar}

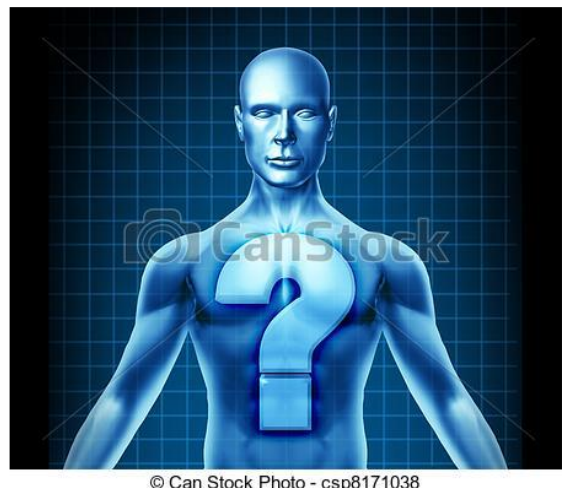

El acto de diagnosticar como competencia, ha sido llamado de distintas formas y una de las más usadas ha sido "el ojo clínico". El principio del diagnóstico es de "sumas y restas". El clínico (el que diagnostica) pregunta, examina y solicita y, con base en encontrar o no encontrar, al final hace su impresión diagnóstica a partir de la cual ofrece su tratamiento, lo que después se confronta con la evolución y decide el destino del acto médico.

Si bien hasta finales del siglo $X X$ se consideró que el diagnóstico se correspondía con el ojo clínico (experiencia) y que, por ende, era muy difícil de enseñar, a raíz del surgimiento de la Medicina Basada en la Evidencia hoy sabemos que se puede enseñar. Precisamente de eso trata el diagnóstico basado en probabilidades, en donde partiendo de la sensibilidad y la especificidad de las manifestaciones de los pacientes, podemos llegar a una probabilidad diagnóstica, expresada en números.

A continuación les mostraremos cómo. Para ello incluiremos un nuevo término que es el cociente de verosimilitud 0 likelihood (positivos y negativos).

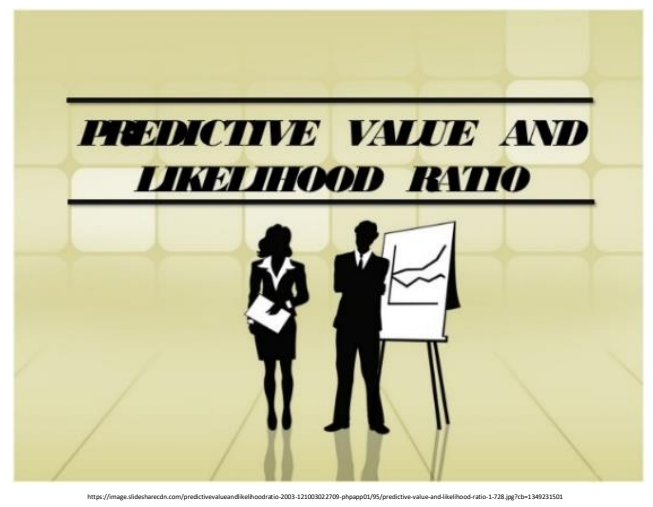

$\rightarrow$ Coeficiente de verosimilitud positivo o Likelihood Ratios (LR+):

\section{Concepto}

Los coeficientes de verosimilitud realizan una comparación en la que se establece la posibilidad de que un resultado dado de una prueba pudiera esperarse en un paciente CON la enfermedad de interés, comparado con la que ese mismo resultado pudiera esperarse en un paciente SIN dicha enfermedad.
Su obtención

Su cálculo se obtiene de dividir la sensibilidad por el complemento de la especificidad.

EI LR (+) se refiere al número de veces en que la prueba es positiva en los enfermos (sensibilidad), sobre el número de veces en que la prueba es positiva en los sanos (falsos positivos o 100 menos la especificidad).

$$
\boldsymbol{L R}(+)=\frac{\text { Sensibilidad }}{100-\text { Especificidad }}
$$


$\rightarrow$ Coeficiente de verosimilitud negativa LR (-):

Se refiere al número de veces en que la prueba es negativa en los enfermos (porcentaje de falsos positivos), sobre el número de veces en que la prueba es negativa en los sanos (especificidad).

$\boldsymbol{L R}(-)=\frac{100-\text { Sensibilidad }}{\text { Especificidad }}$

- Estos dos conceptos y su proceso matemático son claves para la obtención de la probabilidad de enfermedad. iOjo!, de lo que estamos hablando es de que vamos a cambiar esa forma de hacer diagnóstico en la que el médico dice "me parece que es..." por, "la probabilidad es...".

\section{¿Cómo se aplican?}

Recuerden: si continuamos con nuestro ejemplo del electrocardiograma en la enfermedad coronaria, habíamos dicho que tiene una sensibilidad del $43 \%$ y una especificidad del $86 \%$. Calculemos los coeficientes de verosimilitud de acuerdo a las formulas anteriores:

$$
\begin{aligned}
& \boldsymbol{L R}(+)=\frac{\text { Sensibilidad }}{100-\text { Especificidad }}=\frac{43}{100-86}=3,0 \\
& \boldsymbol{L} \boldsymbol{R}(-)=\frac{100-\text { Sensibilidad }}{\text { Especificidad }}=\frac{100-43}{86}=0,67
\end{aligned}
$$

\section{¿Ahora qué hacemos con todos estos datos?}

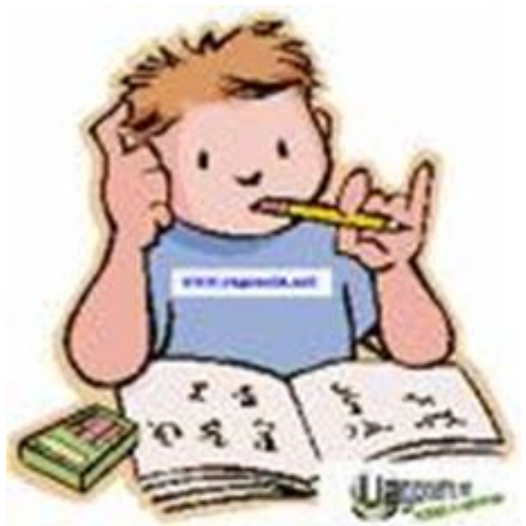

Pues bien ya tenemos suficiente información como para ir progresando en nuestro diagnóstico. Los pasos son como siguen:

$\rightarrow$ Primero: reconocer la prevalencia de la enfermedad de la que sospechamos.

Como lo enunciamos en la introducción, la prevalencia de enfermedad coronaria es del $15 \%$. Utilizaremos este valor como la probabilidad de base de que un paciente que nos llegue al servicio de urgencias tenga enfermedad coronaria y la llamaremos probabilidad preprueba (lo ideal sería que cada uno en su propio sitio de trabajo identifique con un estudio cuál será la probabilidad o la prevalencia de enfermedad coronaria). 
$\rightarrow$ Segundo: aplicar la historia clínica.

Pero continuemos. Un paciente nos llega por dolor torácico y sin haberlo visto ya tenemos una probabilidad del $15 \%$ de que tenga una enfermedad coronaria. Realizamos la historia clínica y nos encontramos que se trata de un paciente de 65 años de edad que refiere dolor precordial irradiado a brazo izquierdo, tipo peso, y a cuello, asociado a diaforesis y vómito.

Pues bien, cada síntoma (indagamos los más característicos de la enfermedad que sospechamos) de nuestro paciente tiene un LR ya sea positivo (si lo tiene) o negativo (si no lo tiene), que vamos a utilizar para calcular el diagnóstico por probabilidades.

\section{¿Cómo lo hacemos?}

Traeremos un nomograma que nos va a facilitar las cosas. Es el llamado nomograma de Fagan. Dicho sea de paso, se trata de una herramienta construida por la estadística, útil para calcular las probabilidades.

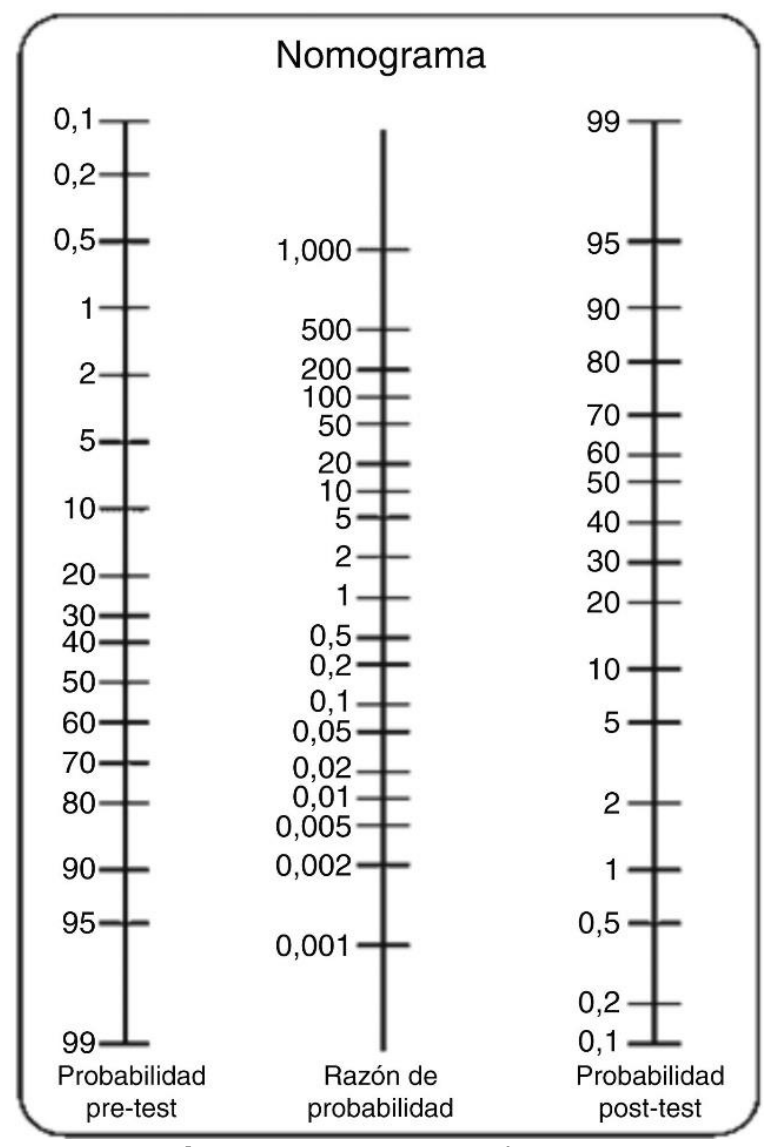

Figura 1. Nomograma de Fagan

Allí vemos tres columnas. La primera dice probabilidad pretest, la segunda dice razón de probabilidades o nuestros LR y la tercera dice probabilidad postest.

\section{Entonces... ¿cómo se usa?}

Iniciemos con la prevalencia de la enfermedad coronaria que, dijimos, es de $15 \%$. Esta será nuestra probabilidad pretest y ubicaremos un punto en la primera columna, que coincida con esta probabilidad, o sea, entre el 10 y el 20 . Ahora tomamos el síntoma dolor precordial típico y nos vamos a la tabla 2 (ver página 61) donde vamos a ubicar el LR (+) que nos arroja un valor de 6,8 . Ubicamos este valor en la columna del centro (en el nomograma) y trazamos una línea que vaya del $15 \%$ hasta el 6,8 y la prolongamos hasta la columna de la derecha. 


\section{¿Qué ocurrió?}

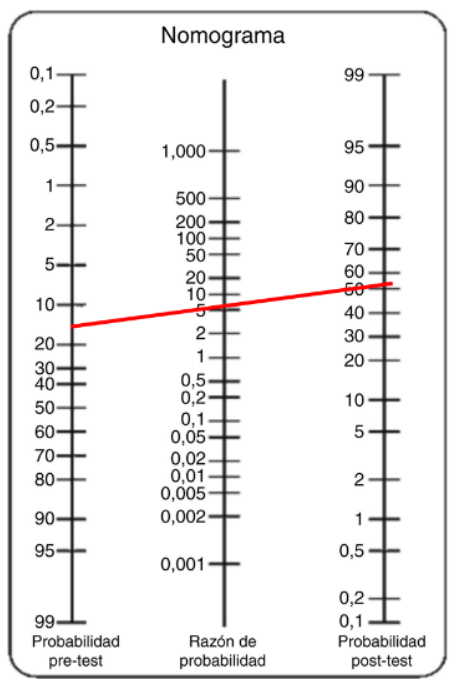

Figura 2

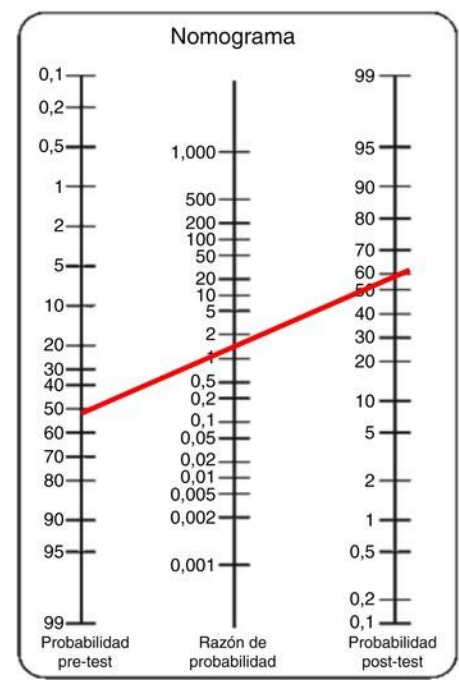

Figura 3

Como vemos la nueva probabilidad postprueba nos queda más o menos en $\mathbf{5 2}$ \% (figura $\mathbf{2}$ ). Es decir, con este paciente pasamos de una probabilidad de enfermedad coronaria del $15 \%$ al $\mathbf{5 2} \%$ por tener el dolor coronario típico, lo que la hace más probable. Pero aún nos deja en la incertidumbre diagnostica.

\section{¿Cómo así que incertidumbre diagnóstica?}

Pues sí. Podemos entenderla como la falta de certeza, cualidad necesaria para tomar las decisiones que conlleven a las intervenciones que creamos beneficiarán al paciente. Por ejemplo, en el caso que estamos analizando, podemos preguntarnos cuál será el porcentaje de incertidumbre (o su contrario, el porcentaje de certeza) que me permitirá decidir iniciar o no lo trombolisis. Una vez más, los números (porcentajes de probabilidad) nos harán más fácil tomar esas decisiones, ¿no les parece?

Entonces, otra vez...

Siguiendo con el ejemplo, ahora la nueva probabilidad pretest nos quedó en $\mathbf{5 2} \%$, la cual ubicaremos ahora en la primera columna del nomograma.
Tomaremos ahora otro síntoma, por ejemplo la diaforesis, que en la tabla nuestra un LR positivo de 1,4, y realizamos el mismo procedimiento (trazar la línea desde esta nueva probabilidad pretest hasta la columna del LR). Como vemos ahora nuestra nueva probabilidad queda en $\mathbf{5 9} \%$.

\section{Así continuamos...}

Con toda la historia del paciente, con los antecedentes y el examen físico, vemos cómo surge la probabilidad diagnostica. Al final de haber analizado los síntomas que tenemos y los signos, incluso los resultados de las pruebas, el nomograma nos arrojara la probabilidad final de que padezca la enfermedad.

\section{Pero, ¿entonces debemos tener de cada enfermedad la tabla de la sensibilidad y especificidad de cada manifestación?}

Así es. De hecho, existen libros donde están analizadas las manifestaciones con su sensibilidad, especificidad y sus respectivos LR (+) y (-). Claro, el ideal es hacer los estudios en cada centro hospitalario y así obtener los "propios" LR. 


\section{Escalas diagnósticas}

Para mejorar nuestra certeza diagnóstica, encontramos en la literatura escalas predictivas validadas que nos ayudan aún más a confirmar o descartar la enfermedad coronaria. Las escalas predictivas están basadas precisamente en la presencia o ausencia de manifestaciones a las que se les pueden calcular LR (+) o LR (-) y, con base en ellas, se puede emitir la probabilidad de la presencia de la enfermedad.

Las escalas de sospecha de enfermedad coronaria más utilizadas son:

ESCALA HEART $(14,15,16,17)$

\begin{tabular}{|c|l|c|}
\hline \multicolumn{2}{|c|}{ ESCALA HEART PARA DOLOR TORÁCICO } \\
\hline \multirow{3}{*}{ HISTORIA } & Alta sospecha. & 2 \\
\cline { 2 - 3 } & Moderada sospecha. & 1 \\
\cline { 2 - 3 } & Baja sospecha. & 0 \\
\hline \multirow{4}{*}{ EKG } & $\begin{array}{l}\text { Depresión significativa del } \\
\text { segmento ST. }\end{array}$ & 2 \\
\cline { 2 - 3 } & $\begin{array}{l}\text { Trastornos difusos de } \\
\text { repolarización. }\end{array}$ & 1 \\
\cline { 2 - 3 } & Normal. & 0 \\
\hline \multirow{3}{*}{ EDAD } & Más de 65 años. & 2 \\
\cline { 2 - 3 } & 45 a 65 años. & 1 \\
\cline { 2 - 3 } RAORES DE & Menos de 45 años. & 0 \\
\hline \multirow{3}{*}{ TROPO } & $\begin{array}{l}\text { Más de tres factores de riesgo o } \\
\text { historia de ateromatosis. }\end{array}$ & 2 \\
\cline { 2 - 3 } & Uno a dos factores de riesgo. & 1 \\
\cline { 2 - 3 } & No factores de riesgo conocidos. & 0 \\
\hline & $\begin{array}{l}\text { Más de tres veces el valor normal } \\
\text { del límite superior }\end{array}$ & 3 \\
\cline { 2 - 3 } & Una a tres veces el valor normal & 2 \\
\cline { 2 - 3 } & Menor de lo normal & 1 \\
\hline
\end{tabular}

La escala HEART (por sus siglas en inglés: History, EKG, Age, Risk factors, Troponin) ha sido validada por varios estudios obteniendo una sensibilidad y especificidad de acuerdo a su puntaje. Sirve para calcular la probabilidad de padecer de enfermedad coronaria.

Las instrucciones para diligenciar esta escala son las siguientes:

a. Historia (History): Si el paciente presenta dolor precordial o lateral izquierdo tipo peso, con irradiación y los síntomas mejoran con la utilización de nitratos sublinguales, se le adjudican 2 puntos. En caso de que la historia tuviera algunos elementos específicos e inespecíficos se adjudica un puntaje de 1 . En ausencia de elementos específicos que sugieran isquemia se adjudica un puntaje de 0 .

b. Electrocardiograma (EKG): Se incluyeron los criterios de Minnesota para su interpretación. Si el electrocardiograma no presenta elevación significativa o depresión significativa del ST o alguna anormalidad se adjudica un puntaje de 0 . En caso de presentar un bloqueo de rama, ritmo de marcapaso, criterios de hipertrofia ventricular izquierda, trastornos de repolarizacion dados por la digoxina o disturbios en la repolarizacion se adjudica un puntaje de 1 . En caso de elevación significativa del ST o depresión del ST en ausencia de bloqueo de rama, hipertrofia ventricular o utilización de digoxina se adjudica un puntaje de 2 .

c. Edad (Age): Se adjudicaron 0 puntos si el paciente tiene menos de 45 años, 1 punto si tiene de 45 a 65 años y 2 puntos si tiene más de 65 años.

d. Factores de riesgo (Risk factors): Se tomaron como factores de riesgo la diabetes mellitus en tratamiento, ser fumador actual o en los últimos 90 días, la historia clínica de hipertensión arterial, la hipercolesterolemia, la historia familiar de enfermedad coronaria, la obesidad o índice de masa corporal mayor a 30 y, la historia clínica de ateromatosis (antecedentes de revascularización miocárdica, infarto agudo de miocardio, evento cerebrovascular, enfermedad arterial periférica). Si el paciente no tiene factores de riesgo se adjudican 0 puntos, si tiene de uno a dos factores de riesgo se adjudica 1 punto $y$, si tiene tres o más factores de riesgo, se adjudican 2 puntos.

e. Troponina (Troponin). De acuerdo a los valores locales de laboratorio, se adjudica 1 punto si el valor de la troponina se encuentra por debajo de lo normal. Si el valor se encuentra entre una a tres veces el valor normal superior de acuerdo al percentil 99, de adjudican 2 puntos. Si el valor se encuentra más de tres veces el valor normal se adjudica un puntaje de 3 . 


\section{Interpretación de la escala HEART}

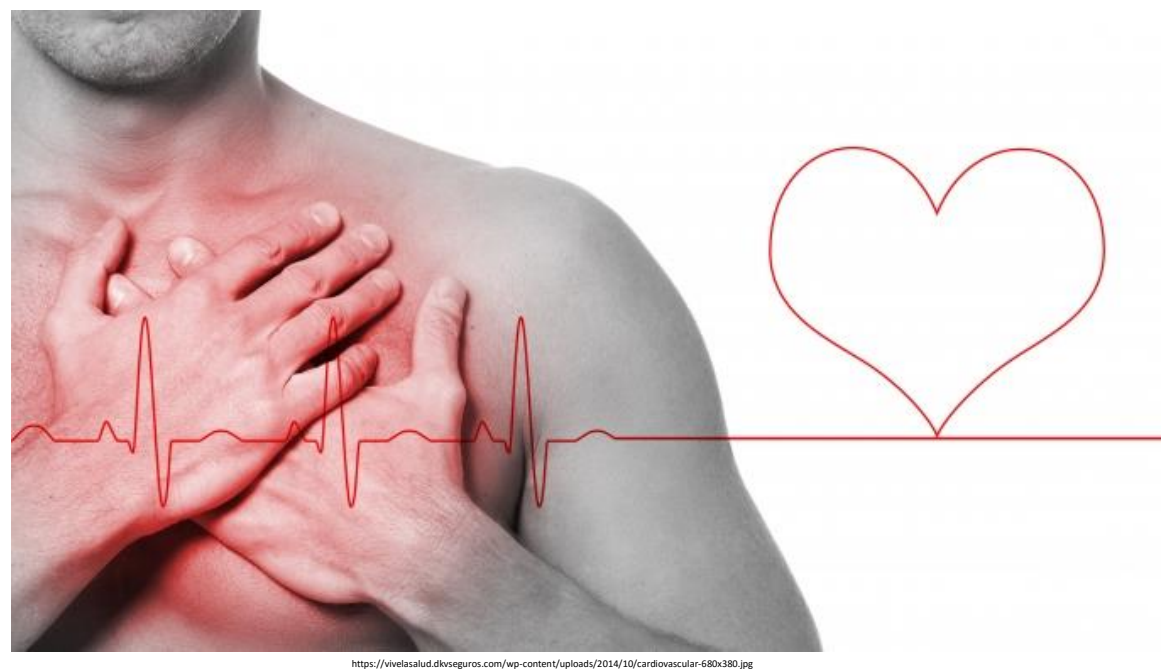

Los puntajes para esta tabla se clasificaron así:

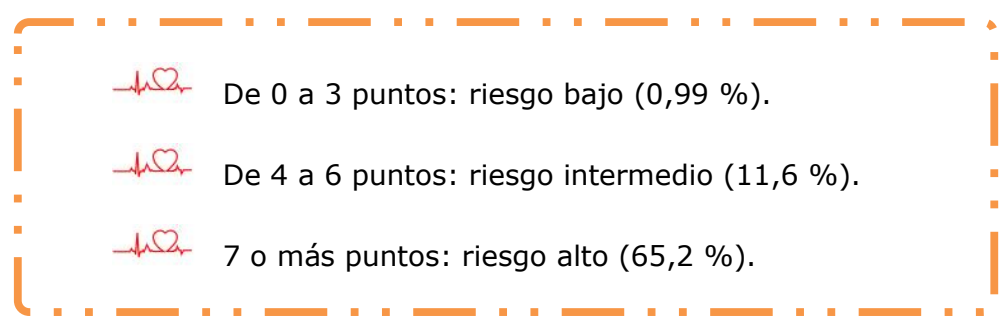

\section{Combinando escalas y probabilidades}

La sensibilidad del score cuando se obtiene un valor de 2 o menos puntos es de $98,9(97,3-99)$ y la especificidad es de 14,7 $(13,4-16,2)$ con un LR positivo de 1,14 y LR $(-)$ de 0,01 . Una escala de 7 o más puntos presenta un LR $(+)$ de $6,8(4-12)$ y, un puntaje de 3 a 6 , tiene un LR $(+)$ de $2,6(1,1-6,3)$ con una sensibilidad de $58 \%(32-81)$ y una especificidad de $85 \%(83-87)$.

\section{Potencializarla con nueva troponina}

Para mejorar aún más la sensibilidad de esta escala, se está recurriendo a la toma de una segunda muestra de troponina, cuando ha salido positiva o en el límite en la primera oportunidad. Si la troponina permanece negativa o disminuye y la escala es de 3 o menos puntos, se puede descartar evento coronario agudo y buscar otra causa.

Al contrario, si la troponina aumenta o se vuelve positiva el paciente debe hospitalizarse para estudio. Si el puntaje es de 7 o más, la probabilidad de enfermedad coronaria es muy alta y este paciente debe también hospitalizarse. Six et al (18) reportaron en su validación de la escala un aumento de la sensibilidad al $100 \%$ y de la especificidad al 83 \% con la seriación de troponinas. 


\section{Escala GRACE (Global Registry of Acute Coronary Syndrome) $(18,19)$}

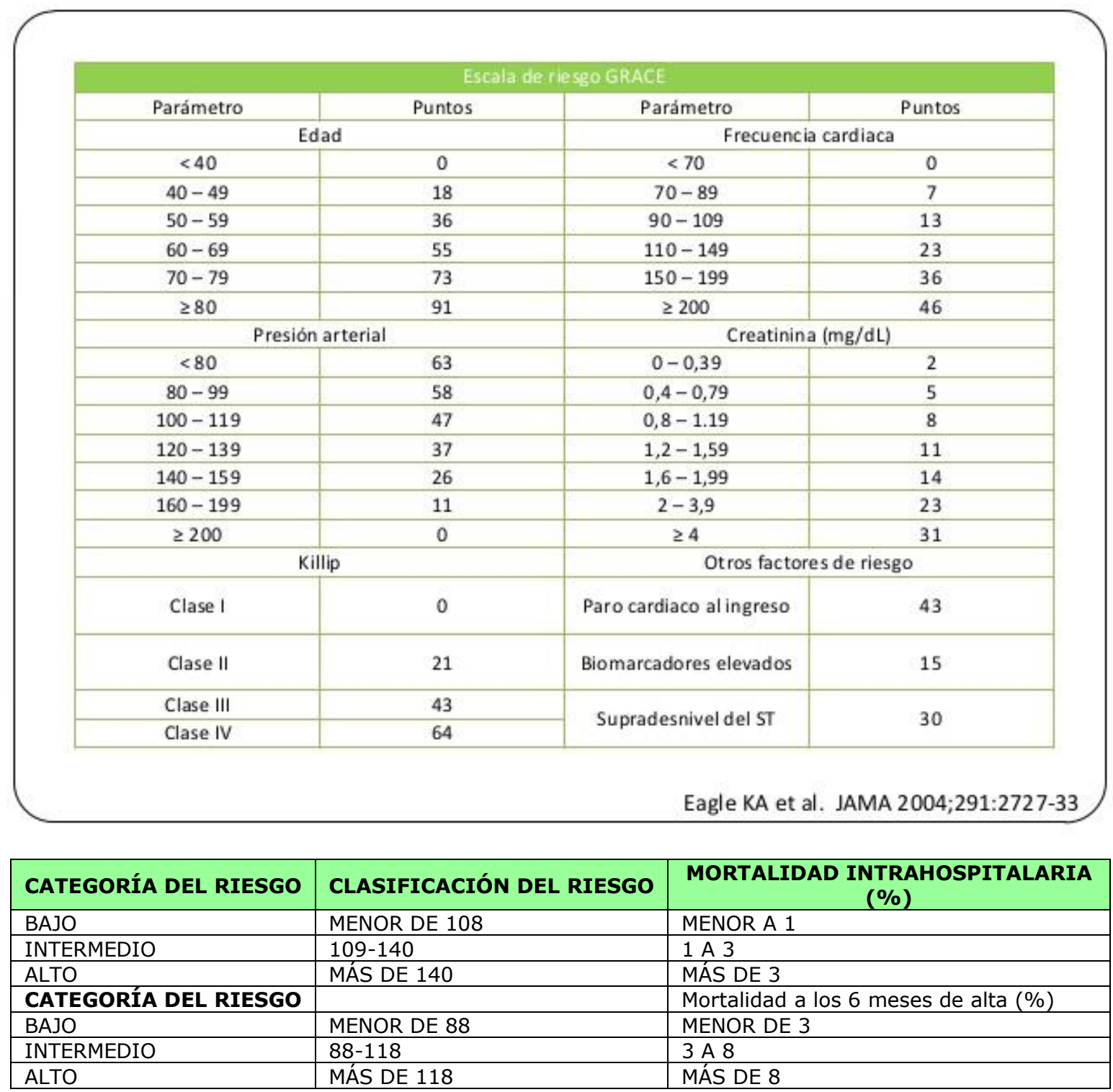

Tal vez en la actualidad la escala más utilizada. Su utilidad radica en la predicción de mortalidad intrahospitalaria y, a los seis meses, del IAM (Infarto Agudo de Miocardio).

\section{¿Cómo se validó?}

La valoración GRACE (GRACE score) fue desarrollada a partir de un registro multinacional (Global Registry of Acute Coronary Syndrome) de 11389 pacientes con SCA (20) durante dos años en 94 hospitales localizados en 18 zonas geográficas y 14 países (Argentina, Australia, Austria, Bélgica, Brasil, Canadá, Francia, Alemania, Italia, Nueva Zelanda, Polonia, España, Reino Unido y Estados Unidos). 
TROPONINAS $(9,21,22,23,24,25)$

\section{Su importancia}

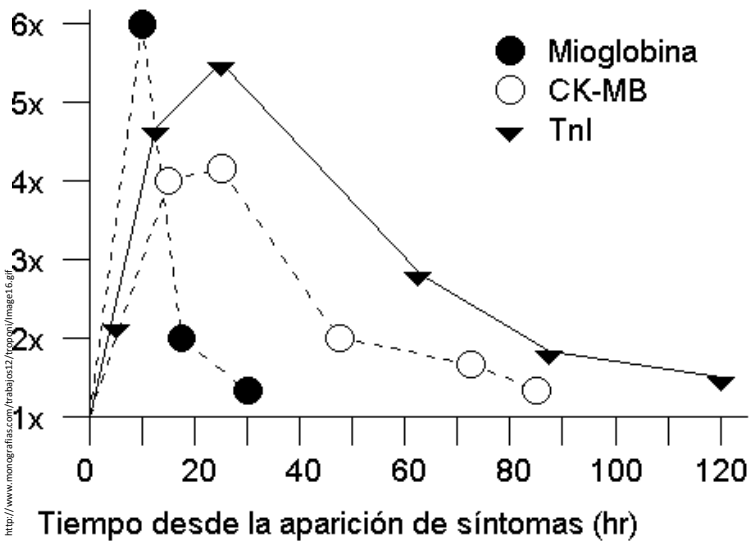

La troponina y el electrocardiograma son dos piedras angulares que apoyan el diagnóstico de enfermedad coronaria, unidos, claro está, a la realización de la historia clínica.

Como vimos, la sensibilidad y la especificidad del electrocardiograma no son lo suficientemente robustas como para afirmar que un determinado paciente presenta o no un evento coronario por su alto índice de falsos positivos (cardiomiopatía hipertrófica, síndrome de Brugada, pericarditis, bloqueo de rama izquierda, patrón juvenil) o por sus falsos negativos.

\section{Por ello}

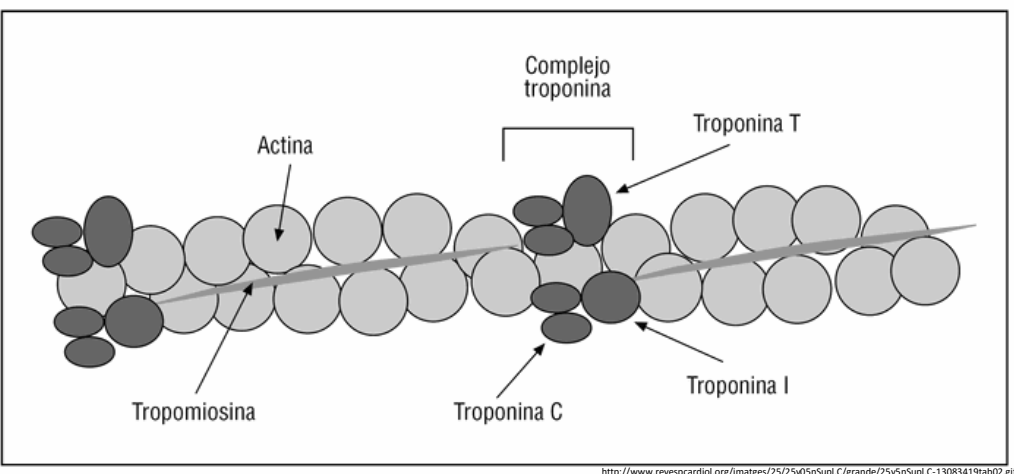

Los llamados biomarcadores han venido evolucionando en los últimos años, mejorando así su sensibilidad y especificidad. Es así como en la década de los años 1960 se utilizaban las transaminasas $\mathrm{y}$, posteriormente, la deshidrogenasa lactina, la mioglobina, la CPK MB y, por luego, las troponinas. Aun así, estas últimas también vienen evolucionando desde las troponinas de primera generación hasta las troponinas ultrasensibles que son las que utilizamos en la actualidad. Pero los problemas aún persisten ya que esta prueba también tiene deficiencias por sus falsos positivos.

\section{Causas de elevación de la troponina (11)}

1. Infarto agudo de miocardio.

2. Taqui o bradiarritmias.

3. Disección de la aorta o, insuficiencia o estenosis aórtica, severas.

4. Hipotensión severa (choque séptico o por hemorragias), emergencias hipertensivas o crisis hipertensivas.

5. Falla cardiaca aguda o crónica.

6. Cardiomiopatía hipertrófica tako-tsubo.

7. Vasculitis coronaria como en el lupus eritematosos sistémico o síndrome de Kawasaki.

8. Espasmo de las arterias coronarias por cocaína.

9. Tromboembolismo pulmonar severo o hipertensión pulmonar severa.

10. Hemodiálisis.
11. Desfibrilaciones frecuentes.

12. Contusión cardiaca o cirugía cardiaca.

13. Rabdomiolisis.

14. Miocarditis.

15. Agentes cardiotóxicos.

16. Quemaduras de más del $30 \%$ de superficie corporal.

17. Eventos cerebrovasculares o condiciones neurológicas severas o trauma craneoencefálico.

18. Enfermedades infiltrarias como sarcoidosis o amiloidosis.

19. Ejercicio extremo (correr maratones, caminatas en exceso, como en los militares).

20. Sepsis, hipotiroidismo o hipertiroidismo. 


\section{Diagnóstico por probabilidades}

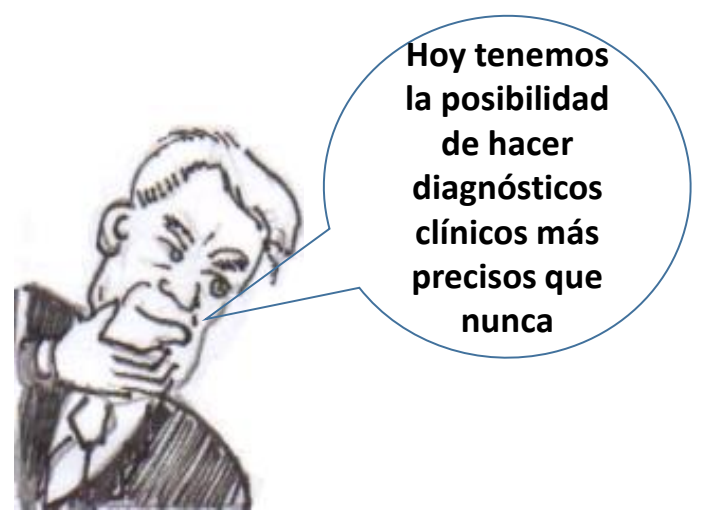

Uniendo todo, vamos a continuar con nuestra descripción basados en la sensibilidad y especificidad, así como el valor de sus LR, para comprender aún mejor estos conceptos.

Tomaremos de ejemplo la tabla realizada en la publicación del New England Journal Medicine sobre la sensibilidad y especificidad de la troponina en el dolor torácico y de ella tomaremos la troponina T de la marca Roche utilizada en nuestra institución (9).

\section{La tabla comparativa}

\begin{tabular}{|c|c|c|c|c|c|}
\hline \multirow{2}{*}{ TROPONINA T } & \multicolumn{4}{|c|}{ INFARTO AGUDO DE MIOCARDIO } & \multirow{2}{*}{ TOTAL } \\
\hline & Sí & & NO & & \\
\hline Positiva & 117 (VP) & a & $119(\mathrm{FP})$ & b & 236 (VPP) $\mathbf{g}$ \\
\hline Negativa & $6(\mathrm{FN})$ & $\mathbf{C}$ & $476(\mathrm{VN})$ & d & 482 (VPN) h \\
\hline TOTAL & 123 & $\mathbf{e}$ & 595 & $f$ & 718 \\
\hline
\end{tabular}

Entonces, repasando...

$\rightarrow \quad$ ¿Cuál es la prevalencia de infarto agudo de miocardio en el estudio?

Si el total de los pacientes fue de 718 y, de estos, 123 tuvieron infarto agudo de miocardio, solo tendremos que obtener qué porcentaje es 123 de 718 . Esto nos da $17,13 \%$.

$\rightarrow \quad$ ¿Cuántos pacientes presentaban infarto agudo de miocardio y la troponina $T$ arrojó un resultado positivo?

La respuesta es 117 . Como se observa corresponde a la letra a.

\section{Sensibilidad}

$\rightarrow$ Observemos la casilla a. De los que presentaron infarto agudo de miocardio, ¿qué porcentaje tenía la prueba de troponina T positiva?

$\rightarrow \quad \mathrm{O}$, lo que es lo mismo, dado que estoy enfermo, ¿qué probabilidad tengo de que la prueba me salga positiva?

\section{Formas de decir lo mismo}

Como vemos, esta pregunta corresponde a la sensibilidad de la prueba. La operación consiste en calcular a qué porcentaje corresponde 117 de 123. En palabras:

$\rightarrow$ De los que presentaban infarto agudo de miocardio (123), ¿qué porcentaje presentó troponina positiva?

$\rightarrow \quad 0$, de los que estaban verdaderamente enfermos (123), ¿a cuántos detectó la prueba como verdaderamente enfermos $(117) ?$

Para esto, se realiza una regla de tres simple y nos arroja un resultado de $95 \%$ que quiere decir que la prueba de cada 100 pacientes enfermos detecta a 95 de ellos, pero se le escapan cinco personas que tenían infarto agudo de miocardio. 
Por ende, y usando las letras de las casillas.

Para obtener la sensibilidad de una prueba en una tabla de $2 \times 2$, realizaremos la siguiente operación: $a /(a+c)$ y esto corresponde a los llamados verdaderos positivos (VP).

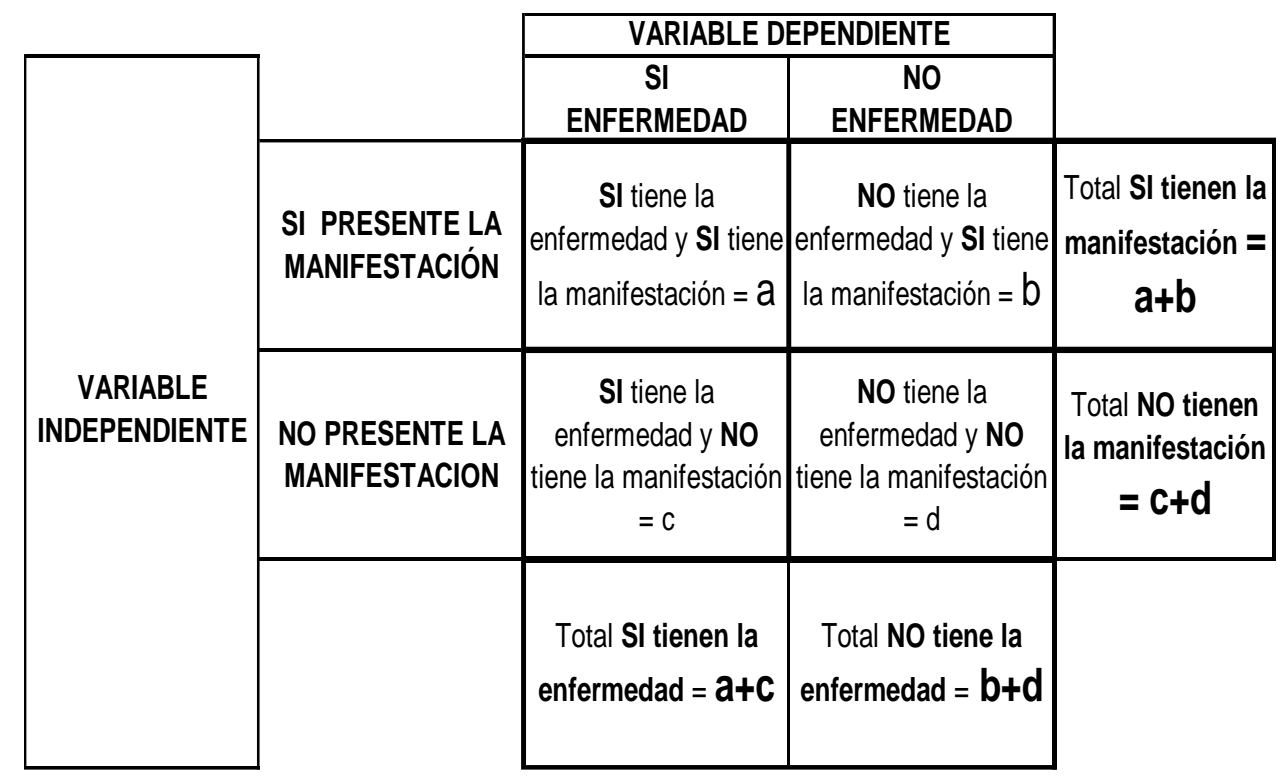

\section{Especificidad}

Dirijámonos ahora a la casilla b, ¿qué ve?

$\rightarrow \quad$ ¿Cuántos pacientes NO presentaban infarto agudo de miocardio, pero la prueba mostró que sí lo tenían, es decir, salió positiva?

$\rightarrow \quad \mathrm{O}$, dado que estoy sano, ¿qué probabilidad tengo de que la prueba me resulte positiva?

Aquí nos referimos al porcentaje de pacientes de la casilla $\mathbf{b}$. Como podemos observar, en esta casilla hay 119 pacientes de los 595 que no presentaban infarto agudo de miocardio, pero la prueba dijo que sí estaban enfermos, estos son los llamados FALSOS POSITIVOS, que, en el ejemplo, corresponden al $20 \%$. Para obtener este cociente realizaremos la siguiente operación: $b /(b+d)$. Como vemos aquí, los resultados de la prueba no coinciden con la condición del paciente, que para este caso no presentaba infarto agudo de miocardio. El problema aquí yace en que a pacientes sin enfermedad coronaria los podremos exponer a métodos diagnósticos innecesarios riesgosos y hasta costosos (vea causas de la elevación de la troponina en la página 70).

\section{Ahora observemos la casilla c}

Aquí la casilla nos indica el número de pacientes que sí presentaban un infarto agudo de miocardio pero la prueba salió negativa. Se llaman los falsos negativos que quiere decir que si mi paciente tiene la enfermedad la prueba no la detecta.

\section{La operación matemática}

Para obtener este valor realizaremos la siguiente operación matemática: $c /(a+c)$ y nos arroja un resultado de 4,87 \%. Aquí se presenta el otro problema ya que, si no interpretamos en conjunto la historia clínica, el electrocardiograma y el resultado de la troponina podríamos enviar a casa a un paciente con infarto agudo de miocardio. 


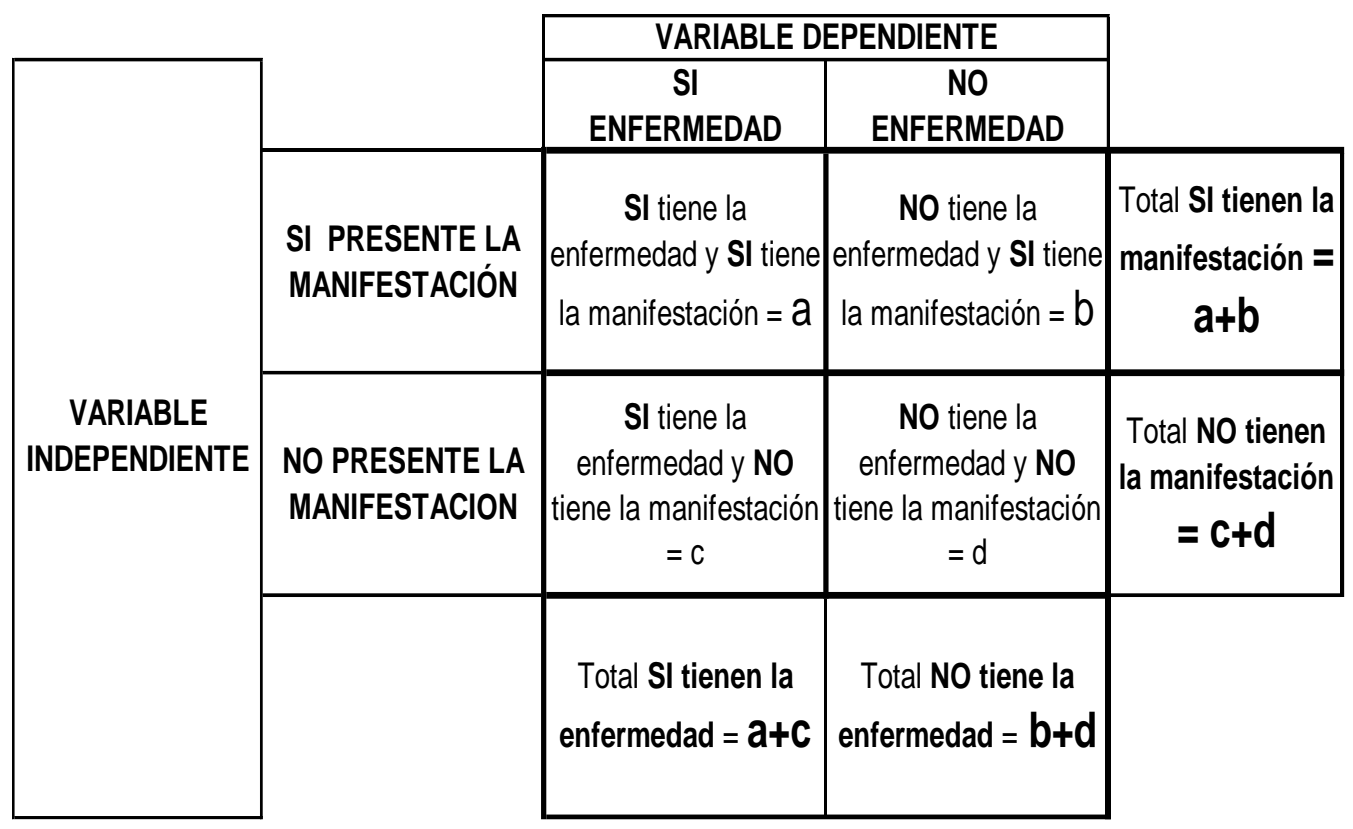

\section{Analicemos la casilla d: especificidad}

La de la llamada especificidad. Esta casilla se refiere a los pacientes que verdaderamente están sanos o, para este caso, no presentan infarto agudo de miocardio y la prueba sale negativa. Es llamada también los verdaderos negativos. Responde a la pregunta, dado que estoy sano, ¿qué probabilidad tengo de que la prueba salga negativa? La operación matemática es la siguiente: $d /(d+b)$ y su resultado es $80 \%$, que quiere decir que, de cada 100 sanos, detecta a 80 y se le escaparán 20 .

\section{Uniendo todo}

Observemos muy bien lo siguiente:

$\rightarrow$ La sensibilidad nos arrojó un resultado del $95 \%$, o sea, los verdaderos positivos y su complemento será la casilla c que nos arrojó 4,87 \%, o sea, los falsos negativos. La suma de estos dos porcentajes debe dar el $100 \%$.

$\rightarrow$ Lo mismo sucede con la especificidad; si vemos, la especificidad fue del $80 \%$ y su complemento, o sea el porcentaje de la casilla b o los falsos positivos, nos arrojó un resultado del $20 \%$. La suma de estos dos porcentajes nos da $100 \%$.

\section{Conclusión}

Recuerde que el complemento de la sensibilidad es el porcentaje de los falsos negativos y el complemento de la especificidad es el porcentaje de los falsos positivos.

\section{LOS VALORES PREDICTIVOS}

Si observamos bien, para obtener la sensibilidad o la especificidad las operaciones matemáticas se realizaron sobre las columnas del Sí y del NO de la tabla de 2 X2 y, su lectura, también va en ese sentido. Ahora vamos a trabajar sobre las filas o en sentido horizontal y con ellas obtendremos los valores predictivos. 
El valor predictivo positivo

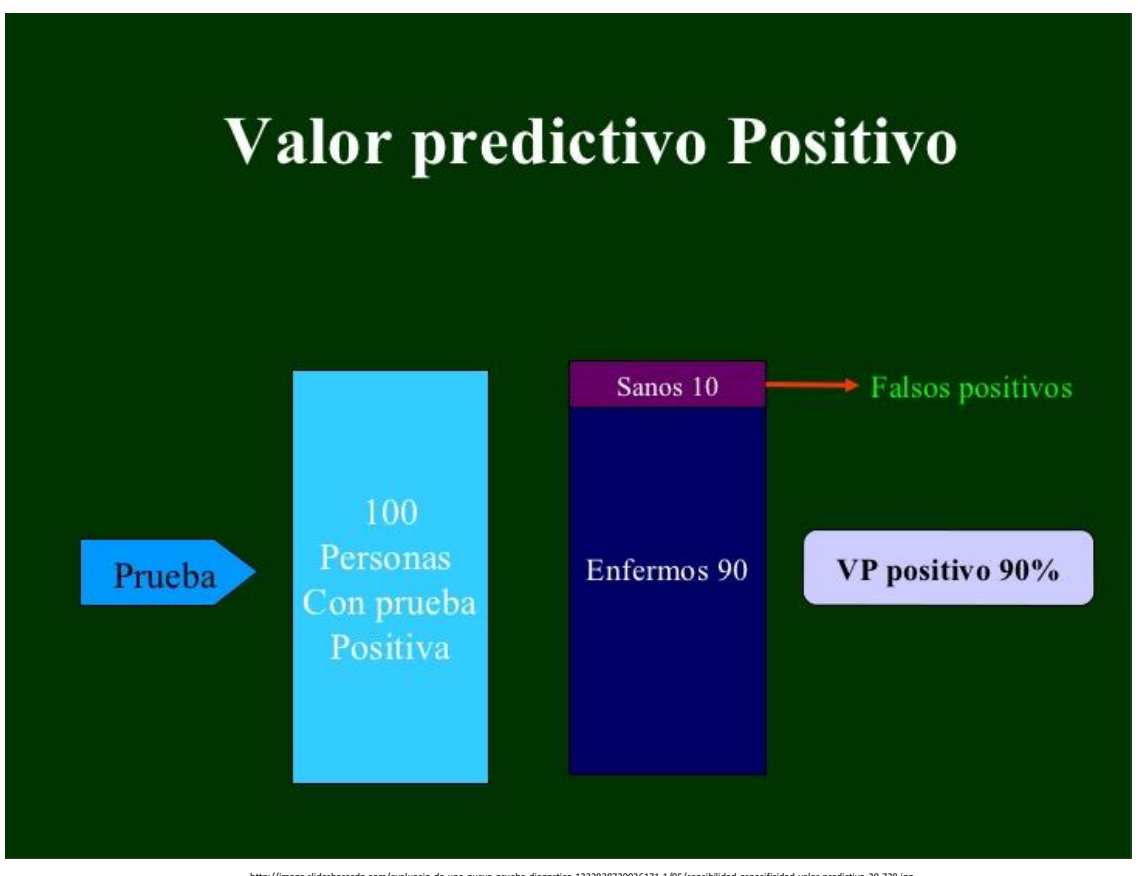

Existen muchas formas de parafrasear el valor predictivo positivo, una de ellas es:

$\rightarrow$ Dado que tengo una prueba positiva, ¿qué probabilidad tengo de estar enfermo?

Que es muy diferente a decir:

$\rightarrow \quad$ Dado que tengo una enfermedad, ¿qué probabilidad tengo de obtener un resultado positivo en una prueba?

\section{Diferenciando a la sensibilidad}

Si nos fijamos bien, la primera, es decir, el valor predictivo positivo, lo leímos en forma horizontal, iniciando nuestra pregunta con la prueba; mientras que en la segunda frase (que por cierto corresponde a la sensibilidad) la lectura fue en sentido vertical y ubicamos primero la condición, o sea, estar enfermo, y luego la prueba. Entendiendo esto la forma matemática de obtener el valor predictivo positivo es: $a /(a+b)$ o VP/ (VP+FP).

\begin{tabular}{|c|c|c|c|}
\hline \multirow{2}{*}{ TROPONINA T } & \multicolumn{2}{|c|}{ INFARTO AGUDO DE MIOCARDIO } & \multirow{2}{*}{ TOTAL } \\
\hline & Sí & NO & \\
\hline Positiva & $117(\mathrm{VP})$ & $119(\mathrm{FP})$ & 236 (VPP) $\mathbf{g}$ \\
\hline Negativa & $6(\mathrm{FN})$ & $476(\mathrm{VN})$ & 482 (VPN) h \\
\hline TOTAL & 123 & 595 & 718 \\
\hline
\end{tabular}

\section{Para el valor predictivo negativo}

Realizaremos lo mismo pero con la fila de la troponina T negativa. Seria así:

$\rightarrow$ Dado que tengo una prueba de troponina T negativa, ¿qué probabilidad tengo de estar sano? 
Diferenciando de la especificidad

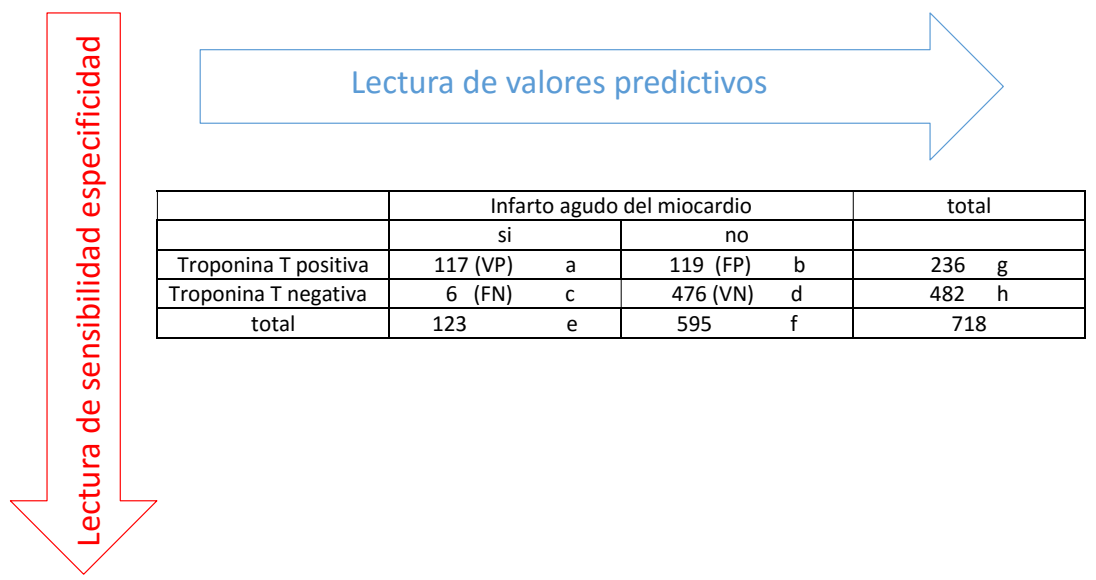

Recuerde que, para la especificidad, la lectura sería en forma vertical:

$\rightarrow$ Dado que estoy sano, ¿qué probabilidad tengo de tener la prueba negativa?

La fórmula matemática para obtener el valor predictivo negativo es: $d /(d+c)$.

\section{Entonces y aplicado}

Para nuestro ejemplo la situación sería la siguiente:

1. Dado que tengo un infarto agudo de miocardio, ¿qué probabilidad tengo de tener una troponina $\mathrm{T}$ positiva? Esto corresponde a la sensibilidad y es del $95 \%$, lo que quiere decir que, de cada 100 pacientes con la enfermedad, la troponina $T$ detecta a 95 de ellos.

2. Dado que tengo una troponina T positiva, ¿qué probabilidad tengo de tener un infarto agudo de miocardio? Este valor correspondería al valor predictivo positivo y nos da como resultado un $50 \%$ de probabilidad.
3. Dado que no tengo infarto agudo de miocardio, ¿qué probabilidad tengo de tener una troponina $T$ negativa? Esto corresponde a la especificidad y su resultado fue del $80 \%$.

4. Dado que tengo una troponina T negativa, ¿qué probabilidad tengo de no padecer un infarto agudo de miocardio? Esto corresponde al valor predictivo negativo y es del 98,7 \% (aproximando $99 \%$ ).

\section{¿Y los valores de verosimilitud o LR?}

Bueno aquí de nuevo los remito a las páginas 63 y 64 para que calculen los valores predictivos de la troponina T con las fórmulas que se explicaron allí.

\section{¿Qué nos queda por hacer?}

Si hemos comprendido el tema, podemos tomar las sensibilidades, especificidades los valores de verosimilitud y el nomograma de Fagan y construir nuestras probabilidades de cada enfermedad con cada uno de nuestros pacientes.

\section{Dónde buscar}

Sugiero revisar la publicación de la revista JAMA, The Rational Clinical Examination, en donde encontrarán un gran número de patologías con sus diferentes síntomas, signos, factores de riesgo, pruebas diagnósticas, etc. con sus respectivos LR que nos ayudarán a depurar cuáles son los síntomas, signos y pruebas diagnósticas más importantes en cada una de ellas. Recuerde que, cuanto más altos sean los LR positivos, nos ofrecen más peso para tener la enfermedad y, que cuanto más bajos sean los LR negativos, más nos ayudarán a descartar la enfermedad. 


\section{REFERENCIAS}

(1) Rosamond W, Flegal K, Furie K, Go A, Greenlund K, Haase N et al. American Heart Association Statistics Committee and Stroke Statistics Subcommittee SO Circulation. Heart Disease and Stroke Statistics-2008 Update: A report from the American Heart Association Statistics Committee and Stroke Statistics Subcommittee. 2008; 117(4):e25.

(2) OPS, Ministerio de Protección Social de Colombia, Instituto Nacional de Salud. Indicadores básicos. Situación de Salud en Colombia. Disponible en: http://www.minsalud.gov.co/salud/paginas/indicadoresbasicossp.aspx

(3) Observatorio Nacional de Salud. Primer Informe ONS. Aspectos relacionados con la frecuencia de uso de los servicios de salud, mortalidad y discapacidad en Colombia, 2011. Bogotá 2013.

(4) Lee TH, Goldman L. Evaluation of the patient with acute chest pain. N Engl J Med 2000; 342 (16): 1187-1195.

(5) Erhardt L, Herlitz J, Bossaert L, Halinen M, Keltai M, Koster R, et al. I Task force on the management of chest pain. Eur Heart J 2002; 23: 1153- 1176.

(6) Uretsky B, Farquhar D, Berezin A y col. Symtomatic Myocardial Infartion Without Chest Pain. Prevalence and clinical course Am J: Cardiology 1077; 40:498-503.

(7) Campeau L. Grading of angina pectoris. Circulation. 1976; 54:522-523.

(8) Guías de práctica clínica basada en la evidencia. ISS-ASCOFAME.

(9) Reichlin R, Hochholzer W, Bassetti S, Steuer S, Stelzig C, Hartwiger S et al. Early Diagnosis of Myocardial Infarction with Sensitive Cardiac Troponin Assays. New England Journal of Medicine. 2009; 361:858-67. doi: 10.1056/NEJMoa0900428.

(10) Hamm CW, Bassand JP, Aquewall S, Bax J, Boersma E, Bueno H et al. ESC Guidelines for the Management of Acute Coronary Syndromes in Patients Presenting Without Persistent ST-Segment Elevation: The Task Force for the Management of Acute Coronary Syndromes (ACS) in Patients Presenting Without Persistent ST-segment Elevation of the European Society of Cardiology (ESC). Eur Heart J. 2011; 32(23):2999-3054. doi: 10.1093/eurheartj/ehr236.

(11) Canto JG, Fincher C, Kiefe CI, Allison JJ, Li Q, Funkhouser E et al. Atypical Presentations Among Medicare Beneficiaries with Unstable Angina Pectoris. Am J Cardiol 2002; 90:248-253.

(12) Thygesen K, Alpert J, Jaffe A, Simoons ML, Chaitman BR, White HD (The Writing Group on behalf of the Joint ESC/ACCF/AHA/WHF Task Force for the Universal Definition of Myocardial Infarction). Third Universal Definition of Myocardial Infarction. Circulation. 2012; 126:2020-2035; originally published online August 24, 2012; doi: 10.1161/CIR.0b013e31826e1058.

(13) Fanaroff AC, Rymer JA, Goldstein SA, et al. Does this Patient with Chest Pain Have Acute Coronary Syndrome? The Rational Clinical Examination Systematic Review. JAMA. doi:10.1001/jama. 2015.12735.

(14) Backus BE, Six J, Kelder JC, Mast TP, Akker F, Mast EG et al. Chest Pain in the Emergency Room. A Multicenter Validation of the HEART Score. Critical Pathways in Cardiology. 2010; 9(3).

(15) Roffi M, Patrono C, Collet JP, Mueller C, Valgimigli M, Andreotti F et al Authors/Task Force Members. ESC Guidelines for the Management of Acute Coronary Syndromes in Patients Presenting without Persistent STsegment Elevation. European Society of Cardiology (ESC). European Heart Journal Advance Access published November 17, 2015.

(16) Mahler SA, Riley RF, Hiestand BC, Russell GB, Hoekstra JW, Lefebvre CW, et al. The HEART Pathway Randomized Trial: Identifying Emergency Department Patients with Acute Chest Pain for Early Discharge. Circ Cardiovasc Qual Outcomes. 2015; 8(2):195-203. doi: 10.1161/CIRCOUTCOMES. 114.001384.

(17) Backus BE, Six AJ, Kelder JC, Bosschaert MAR, Mast EG, Mosterd A et al. A prospective validation of the HEART score for chest pain patients at the emergency Department. Int J Cardiol. 2013; http://dx.doi.org/10.1016/j.ijcard.2013.01.255

(18) Six J, Cullen L, Backus BE, Greenslade J, Parsonage W, Aldous S et al. The HEART Score for the Assessment of Patients with Chest Pain in the Emergency Department. A Multinational Validation Study. Critical Pathways in Cardiology. 2013; 12(3).

(19) GRACE Investigators. Prediction of Risk of Death and Myocardial Infarction in the Six Months after Presentation with Acute Coronary Syndrome: Prospective Multinational Observational Study. BMJ 2006; 333:1091. DOI: http://dx.doi.org/10.1136/bmj.38985.646481.55.

(20) GRACE Investigators. Rationale and design of the GRACE (Global Registry of Acute Coronary Events) Project: A Multinational Registry of Patients Hospitalized with Acute Coronary Syndromes. Am Heart J. 2001; 141(2):190-9. Disponible en: https://www.ncbi.nlm.nih.gov/pubmed/11174331.

(21) Januzzi JL et al. Hs-TnT in chest pain using Cardiac CT. Circulation 2010; 121:1227-1234.

(22) Thygsen $\mathrm{K}$ et al. Universal definition of Acute Myocardial Infarction.Circulation 2007; 116:2634-53.

(23) Thygesen $\mathrm{K}$ et al. ESC Recommendations for troponin use.Eur Heart J. 2010; 3rd August.

(24) Jacobs LH et al. Haemodialysis and hs-TnT. Ann Clin Biochem 2009; 46:283-290.

(25) Jones G. High Sensivity Troponin T. Department of Chemical Pathology Disponible en: http://www.sydpath.stvincents.com.au/tests/ChemFrames/TnT.htm.

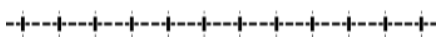

